\title{
LA THÉORIE DE L'ATTACHEMENT : SON IMPORTANCE DANS UN CONTEXTE PÉDIATRIQUE
}

Susana Tereno, Isabel Soares, Eva Martins, Daniel Sampaio, Ellizabeth Carlson

Médecine \& Hygiène | « Devenir »

2007/2 Vol. 19 | pages 151 à 188

ISSN 1015-8154

Article disponible en ligne à l'adresse :

https://www.cairn.info/revue-devenir-2007-2-page-151.htm

\section{Pour citer cet article :}

Susana Tereno et al., " La théorie de l'attachement : son importance dans un contexte pédiatrique », Devenir 2007/2 (Vol. 19), p. 151-188.

DOI 10.3917/dev.072.0151

Distribution électronique Cairn.info pour Médecine \& Hygiène.

(C) Médecine \& Hygiène. Tous droits réservés pour tous pays.

La reproduction ou représentation de cet article, notamment par photocopie, n'est autorisée que dans les limites des conditions générales d'utilisation du site ou, le cas échéant, des conditions générales de la licence souscrite par votre établissement. Toute autre reproduction ou représentation, en tout ou partie, sous quelque forme et de quelque manière que ce soit, est interdite sauf accord préalable et écrit de l'éditeur, en dehors des cas prévus par la législation en vigueur en France. Il est précisé que son stockage dans une base de données est également interdit. 
La théorie de l'attachement: son importance dans un contexte pédiatrique

\author{
Attachment theory: \\ its importance within a pediatric framework
}

Susana Tereno ${ }^{1,2}$, Isabel Soares ${ }^{1}$, Eva Martins ${ }^{1,2}$,

Daniel Sampaio ${ }^{3}$ et Ellizabeth Carlson 4

\section{Théorie de l'attachement}

La thèse, maintenant classique, développée par John Bowlby en 1958, lors de sa première formulation de la théorie de l'attachement, est que l'attachement du bébé à sa figure d'attachement a pour base un équipement comportemental constitué par un nombre déterminé de «réponses instinctives» qui l'orientent vers la figure d'attachement. Bowlby a identifié cinq de ces réponses, qui apportent une contribution spécifique au développement de l'attachement de l'enfant: sucer, attraper, suivre (comportements de proximité ), pleurer et sourire (comportements de signalisation). Ces comportements deviennent intégrés et dirigés vers la figure d'attachement au long de la première année de vie, donnant lieu à ce qu'il a désigné comme les comportements d'attachement. En 1969, Bowlby présente une version reformulée de sa thèse initiale, en reconnaissant que des formes plus élaborées de comportements d'attachement peuvent s'organiser dans un système comportemental d'attachement (Soares, 1996).

Le comportement d'attachement, dans ce sens, est conçu comme une forme de comportement, simple ou organisé, qui aboutit à la recherche ou au maintien de la proximité à un individu différencié et préféré. Lorsque cette figure d'attachement paraît disponible, le comportement peut se limiter à une vérification visuelle ou auditive dirigée vers cette figure, et à un échange occasionnel de regards. Cependant, dans certaines circonstances, l'enfant peut s'adonner à la recherche de la figure d'attachement, ou chercher à l'attraper, de façons à éliciter chez elle un comportement de soins (c.-à-d.: careseeking role). Le comportement symétrique du comportement d'attachement, avec une fonction complémentaire

\section{Département de Psycho- logie, Université du Minho, Portugal. stereno@iep.uminho.pt \\ 2 Boursière de Doctorat par la Fondation pour la Science et la Technologie, Portugal. \\ 3 Faculté de Médicine, Université de Lisboa, Portugal.}

4 Institute of Child Development, Université du Minnesota, USA. 
de protéger l'individu attaché, est celui de prodiguer les soins (c.-à-d.: caregiving role). Tel est le rôle du caregiver ou d'un autre adulte; il est dirigé vers un enfant ou un adolescent, mais il peut aussi être mis en évidence chez un adulte; vis-à-vis d'un autre adulte, spécialement en temps de maladie, de stress, ou d'âge avancé (Bowlby, 1980; Ainsworth, 1985). Durant l'enfance, la proximité physique et la disponibilité émotionnelle du caregiver sont des facteurs critiques de la qualité du caregiving. De la deuxième enfance à la vie adulte, le fait de savoir que le caregiver est potentiellement disponible devient progressivement aussi efficace que la proximité.

L'attachement se réfère au lien émotionnel spécifique que le bébé développe avec son caregiver pendant la première année de sa vie (Bowlby, 1969/1982; 1988). Il se distingue aussi du lien que le caregiver a avec l'enfant et aux processus de liaison qui sont associés aux premières heures de la vie (c.-à-d.: bonding). L'attachement se réfère à la relation qui émerge au fil du temps à partir d'une histoire d'interactions de caregiving $^{5}$ (Sroufe et Waters 1977). Cette relation peut ne pas être présente dans les premières semaines de vie, vu les limitations du bébé dans la compréhension de soi et des autres. Au contraire, à mesure que les adultes interagissent et s'occupent du bébé pendant la première année de la vie, l'enfant organise son comportement autour de ce caregiver.

Le long développement de l'attachement de l'enfant envers sa figure d'attachement est une adaptation évolutive essentielle de l'espèce humaine, dans la mesure où les humains, comme les autres primates, naissent vulnérables, mais le restent durant plusieurs années (Hrdy, 2000). A la lumière d'une perspective phylogénétique, il a été proposé que le système d'attachement ait évolué pour garantir que les petits enfants et les donneurs de soins soient en proximité physique, de façons à assurer la protection des plus jeunes. La figure d'attachement fonctionne, en principe, comme un havre de sécurité, une source de réconfort et de protection dans un contexte d'activation physiologique ou de menace environnementale, et comme base de sécurité pour l'exploration ${ }^{6,7}$ (Bowlby, 1969/ 1982; Ainsworth, 1969; Ainsworth, Blehar, Waters, et Wall, 1978). Ainsi, dans cette perspective, tous les enfants ont tendance à établir des liens avec des adultes qui s'occupent d'eux et qui leurs sont proches, indépendamment du type de traitement qu'ils reçoivent (Bowlby, 1969/1982; Sroufe, 1986). Les études éthologiques et humaines suggèrent que l'attachement se développe même dans un contexte de mauvais traitements et de punitions sévères (Ainsworth, 1967; Belsky et Nezworsky, 1988;

\footnotetext{
5 Les notes se trouvent en fin
} de texte, p. 175 et suivantes. 
Carlson, Cicchetti, Burnett, et al., 1989; Crittenden, 1981; Egelend et Sroufe, 1981; Harlow et Harlow, 1965). Dans ces circonstances, c'est la qualité de cette relation d'attachement qui est en cause, et pas sa présence ou son intensité (Sroufe et Waters, 1977; Ainsworth, 1972).

Les enfants développent d'abord des relations d'attachement avec une figure d'attachement primaire, figure qui s'insère dans son contexte de caregiving (Bowlby, 1969/1982; Ainsworth, 1972; Rutter, 1981); les jeunes enfants peuvent ensuite établir une hiérarchie de ces figures d'attachement, quand il existe plusieurs personnes s'occupant du bébé. Les bébés montrent des comportements d'attachement (p. ex.: pleurer, s'approcher) et obtiennent de la sécurité à partir de figures d'attachement secondaires quand les figures primaires ne sont pas disponibles (Harlow, 1963). Cependant, en réponse à un stress important, leur préférence devient claire, avec la nécessité d'une plus grande proximité avec la figure d'attachement primaire. Une fois établi, ce type de relation préférentielle avec la principale figure d'attachement a tendance à se maintenir, et un changement de comportement d'attachement vers une nouvelle personne ou un étranger devient plus difficile. Dans un réseau de personnes s'occupant de l'enfant (mère, père, grand-père), la qualité de l'attachement est spécifique de la relation en cause, et se construit au travers des interactions avec chacune de ces personnes (Easterbrooks, 1989).

La disponibilité de la figure d'attachement s'accompagne du développement d'une expérience par l'enfant d'une sécurité de l'attachement: l'expérience de la sécurité est associée à une perception de la figure d'attachement comme disponible, et l'anxiété ou l'insécurité est associée à la perception d'une menace face à cette disponibilité.

\section{Le développement des relations d'attachement}

Le contexte de vie d'un enfant est une composante absolument déterminante pour le développement des systèmes comportementaux permettant la formation de l'attachement. Théorisant sur ces dénominateurs communs, Bowlby indique - "When he is born, an infant is far from being a tabula rasa» (1969/1982, p. 265). Au contraire, le bébé est né équipé avec un nombre de systèmes prêts à être déployés lors des stimulations environnementales. Comme nous l'avons mentionné précédemment, quelques-uns de ces systèmes comportementaux constituent les piliers de sustentation du développement de l'attachement, parmi lesquels nous soulignons l'acte de pleurer, d'attraper, la succion, et l'orientation du nouveau-né. 
Bowlby a postulé que le système de développement de l'attachement se construit en quatre phases, les trois premières se déroulant durant la première année de vie et la quatrième commençant autour du troisième anniversaire de l'enfant.

- Phase I - Orientation et signaux avec une discrimination limitée des figures ( 8 à 12 semaines) : le bébé, n'étant pas capable de différencier les personnes (sinon au niveau olfactif et auditif), a tendance à s'orienter de préférence vers les humains en général. Cette tendance, qui promeut et augmente la proximité et le maintien du contact avec ceuxci, se remarque au travers de comportements instinctifs. L'intensité de ces manifestations comportementales atteint son maximum de sociabilité à douze semaines, puisque c'est le moment où les enfants arrivent déjà à reconnaître la figure humaine et montrent un sourire social intentionnel (Ainsworth, et al., 1978).

- Phase II - Orientation et signaux dirigés vers une (ou plusieurs) figure(s) différenciée(s) (12 à 24 semaines): on assiste à l'apparition d'une réponse différenciée du bébé centrée sur la(les) figure(s) avec lesquelles il commence à établir une relation particulière. Cette phase n'a pas un début très clair, parce que la différenciation des comportements est progressive et en fonction de certaines figures, et peut se terminer au-delà de six mois selon les circonstances.

- Phase III - Maintien de la proximité avec une figure différenciée par locomotion et d'autres signaux (6 à 36 mois). Cette période est caractérisée par des avancées significatives, au niveau du développement moteur, cognitif et socio-émotionnel, dans l'organisation des systèmes comportementaux. Les nouvelles compétences, principalement la locomotion, permettent au bébé une exploration plus intense de son entourage. Une plus grande activation du système d'exploration, à cause de l'augmentation du risque qu'elle comporte pour la sécurité du bébé, rend impérative la structuration du système d'attachement, qui vise à protéger l'enfant.

- Phase IV - Formation d'un partenariat réciproque corrigé quant au but (après 36 mois). Graduellement, on assiste à l'abandon d'une position égocentrée de la part de l'enfant, qui aboutit au moment où il commence à être capable de prendre en compte le point de vue de la figure d'attachement. En conséquence, il peut commencer à penser que ses sentiments et ses motivations, ses buts et ses plans peuvent 
influencer le comportement de sa figure d'attachement. Dans ce sens, on parle d'un système corrigé quant au but. L'enfant, recourant à une sorte de carte mentale encore élémentaire, commence à concevoir la figure d'attachement comme une personne indépendante, permanente dans le temps et l'espace, se mouvant dans un continuum spatial et temporel de façon plus ou moins prévisible. La qualité de ces expériences interactionnelles (particulièrement au niveau du langage), ainsi que les compétences acquises vers la décentration du point de vue de l'enfant, sont en relation avec le succès ou l'échec à soutenir la proximité et la communication avec les figures d'attachement ${ }^{8}$ (Soares, 1996).

Nous pouvons alors concevoir que le développement de l'attachement humain se crée à travers une matrice organisée autour du système de soins de la figure parentale, s'exprimant sous la forme de patterns de régulation dyadiques, dans lesquels le degré de participation de l'enfant est progressivement croissant (Sroufe, 1990).

Dans ce contexte, nous pouvons affirmer que, d'après la théorie de l'attachement, pendant les premières semaines de vie déjà, les bébés initient, soutiennent et mettent fin à des interactions sociales (Emde, 1989), bien qu'ils le fassent de façon réflexe, sans intention volontaire. Par exemple, ils se retournent en entendant le son de la voix, prêtant plus d'attention au visage humain et à ses expressions faciales, et ils se calment lors du contact humain (Emde, 1989; Ainsworth, Bell, et Stayton, 1974). Les capacités des enfants, en elles-mêmes, sont toutefois insuffisantes, car la réponse du caregiver est indispensable pour assurer l'autorégulation du bébé (Emde, 1989; Berger, 1974; Koop, 1982). Les expériences relationnelles du bébé avec sa figure d'attachement primaire s'accroissent progressivement pendant les premiers mois, permettant aussi la maturation des processus cognitifs et la synchronisation au niveau neuronal des liens entre les segments de l'expérience et les attentes du bébé (Cicchetti, Ganabian, et Barnett, 1991; Collins et DePue, 1992; Greenough et Black, 1992; Kraemer, 1992; Schore, 1994; Siegel, 2001).

De la naissance jusqu'à 3 mois environ, les modèles régulateurs de l'équilibre physiologique s'établissent et s'organisent dans le contexte de la matrice relationnelle primaire. Par essais et erreurs, le caregiver apprend à interpréter et à répondre de façon adaptée aux états fluctuants et aux signaux de l'enfant (p. ex.: aux pleurs). Ces modèles initiaux de régulation physiologique dans le cadre de la relation de soins servent de prototype pour une régulation psychologique ultérieure (Sroufe, 1995). 
Pendant le deuxième trimestre du bébé, le parent augmente la variété, la complexité comportementale de ses échanges affectifs avec l'enfant, en coordonnant ses expressions faciales, ses vocalisations et la présentation d'objets aux réponses et signaux du bébé selon l'expérience qu'il a acquise lors des séquences d'interaction antérieures. Ces modèles d'interactions permettent à l'enfant de maintenir son niveau d'organisation face à l'augmentation de ses niveaux d'activation physiologique (Brazelton, Koslowsky, et Main, 1974; Sander, 1975; Stern, 1974). L'enfant peut initier une activité et participer dans les interactions orchestrées par le caregiver, mais ne peut pas accomplir ou soutenir cette organisation de façon indépendante. Une fois développée, la répétition de ces comportements et de ces échanges affectifs établissent le fondement des interactions initiées par les bébés, lesquelles sont caractéristiques de la prochaine phase d'organisation de l'attachement (Sander, 1975; Sroufe, 1989).

Le développement psychomoteur de l'enfant facilite le troisième niveau d'organisation dyadique: avec l'émergence de la locomotion, le bébé devient plus efficace dans le maintien de la proximité avec la figure d'attachement (Bowlby, 1969/1982). Avec l'augmentation de sa capacité d'agir de façon intentionnelle, l'enfant assume un rôle plus actif dans l'initiation, le maintien et l'extension de ses modèles comportementaux: il essaie par exemple de suivre le caregiver si celui-ci s'absente, et lui sourit ou lui fait signe s'il revient (Bowlby, 1969/1982; Sroufe, 1989). A partir des interactions orchestrées par le caregiver émerge une relation d'attachement spécifique, caractérisée par la réciprocité (Sroufe, 1989).

Pendants les derniers mois de la première année, l'organisation comportementale du bébé reflète son rôle actif dans le système dyadique. Le donneur de soins assume le rôle de havre de sécurité ou de base de sécurité (Ainsworth, 1973) autour duquel l'enfant centre ses activités exploratrices. Les différences individuelles entre les modèles de régulation dyadiques deviennent plus évidentes. Ces variations reflètent des différences dans l'histoire du mode de caregiving et dans les expectatives de l'enfant concernant la disponibilité de la figure d'attachement.

Dans des circonstances de soins adéquats et sensibles, l'enfant, quand il est effrayé ou en souffrance, a recours à la figure d'attachement, étant donné que celle-ci, reconnaissant l'expression de ses besoins, lui offre du réconfort jusqu'à ce qu'il se tranquillise à nouveau. Au travers des interactions de ce type et à travers le partage d'émotions positives, l'enfant développe la confiance relative à la disponibilité de la figure d'attache- 
ment et à l'efficacité de ses propres initiatives. C'est ce mécanisme qui caractérise une relation d'attachement «sécure». Les relations avec un moindre degré de confiance (attachement anxieux ou insécure) sont associées à des soins marqués par l'indisponibilité, l'inconsistance de la proximité physique ou émotionnelle de la figure d'attachement ou par le rejet répété des signaux et comportements de l'enfant, en particulier dans les situations de stress.

\section{Les Patterns d'attachement}

Selon Bowlby (1969/1982), le développement favorable de l'attachement étant important pour la santé mentale, il devient impératif de distinguer un développement favorable d'un développement défavorable, et de connaître quelles conditions favorisent l'un ou l'autre. En se basant sur la perspective du développement de l'attachement, nous pouvons faire deux hypothèses pour atteindre ces objectifs: 1) les différences relatives à la qualité des soins (régulation par la figure d'attachement) entraînent des différences dans la qualité de l'attachement (régulation dyadique);2) les différences dans les patterns d'attachement vont influencer l'autorégulation émotionelle de l'individu. Dans cette section, nous chercherons à analyser particulièrement la contribution de Mary Ainsworth.

Mary Ainsworth a beaucoup contribué à l'apport des études de l'attachement, notamment en trois domaines: a) le concept de base de sécurité; b) l'importance du rôle de la figure d'attachement; et c) la notion de sensibilité maternelle ${ }^{9}$ aux besoins et aux signaux du bébé.

La notion de base de sécurité désigne le fait pour la figure d'attachement de représenter un support à partir duquel le bébé peut explorer le monde avec confiance. La perception intérieure d'une relation sécure avec la figure d'attachement fonctionne comme un ancrage qui permet au bébé d'activer son système d'exploration. Dans cette ligne d'idée, la sécurité dans l'attachement est définie comme un état de confiance quant à la disponibilité de la figure d'attachement (Ainsworth, et al., 1978). D'après Soares (2002), l'investigation d'Ainsworth et al. a mis en évidence que le modèle de sécurité fournie par la figure d'attachement dans un contexte relationnel se transforme en confiance dans la relation du «self» avec la figure d'attachement et, postérieurement, en confiance dans le «self». C'est dans ce sens que l'auteur conçoit l'espace relationnel comme organisateur de l'attachement.

Cette hypothèse appelle une évaluation validée des patterns d'attachement, laquelle a été opérationnalisée au travers du procédé de laboratoire connu sous le nom de Situation Etrange. 
Mary Ainsworth et ses collaborateurs ont développé ce type de procédé sur la base d'une recherche de terrain en Ouganda (Ainsworth, et al., 1973, 1978) et d'observations réalisées dans le contexte familier au long de la première année de vie aux Etats-Unis (Ainsworth, et al., 1978). Suite à cette étude longitudinale et aux observations à domicile, les mères et les bébés ont été observés en laboratoire avec le paradigme expérimental de la Situation Etrange, conçu pour évaluer l'organisation de l'attachement et le comportement exploratoire dans un contexte non familier et générateur d'un niveau modéré de stress. La Situation consiste en une série de huit épisodes, qui incluent des séparations et des réunions entre l'enfant et sa figure d'attachement, ainsi que l'introduction d'un adulte étranger dans la pièce d'observation.

L'évaluation de la qualité des relations d'attachement se base sur la fréquence et la durée des comportements interactifs entre l'enfant et la figure d'attachement pendant les épisodes de réunion, après une brève séparation (recherche de proximité, maintien du contact, résistance au contact). Les enfants sont classés dans une de ces quatre catégories: groupe A: attachement insécure évitant; groupe B: attachement sécure; groupe $\mathrm{C}$ : attachement insécure ambivalent ou résistant (Ainsworth, et al., 1978); ou groupe D: attachement désorganisé/désorienté (Main et Solomon, 1990). La validité et la fiabilité de ce procédé ont été démontrées pour les enfants d'âges compris entre 12 et 18 mois; la fiabilité et la fidélité de la cotation exigent une formation et de l'entraînement (Carlson et Sroufe, 1993) ${ }^{10}$.

Dans la Situation Etrange, un attachement sécure se reflète par l'utilisation active et la confiance de l'enfant envers la figure d'attachement pour réguler ses émotions (Sroufe et Waters, 1977). Ces enfants se laissent facilement aller à l'exploration quand ils sont en présence de cette figure d'attachement et ne se méfient pas excessivement de l'adulte étranger. Quand ils sont menacés ou angoissés par la séparation, ils signalent leur besoin directement, cherchant activement la proximité et le contact avec la figure d'attachement, qui apparaît pour eux presque immédiatement tranquillisante. Les émotions négatives (angoisse ou colère), exprimées durant la séparation ou pour communiquer ses besoins au caregiver, n'interfèrent pas avec l'exploration renouvelée et le partage d'émotions positives avec la figure d'attachement.

Les enfants avec une relation d'attachement insécure évitant paraissent se confronter à l'augmentation de l'activation physiologique induite en situation de laboratoire par la mise en jeu d'un contrôle excessif de 
l'émotivité (Main, 1981). Ils se lancent dans l'exploration, en interagissant affectivement très peu avec la figure d'attachement, en ne partageant pas l'enthousiasme pour un jouet ou pour un succès, et en faisant preuve d'une discrimination minime dans le traitement de la figure d'attachement et de la figure étrangère. Plus notable encore est le fait que ces bébés n'initient pas activement l'interaction ou la recherche de contact avec la figure d'attachement après la séparation, et que les émotions négatives (la colère par exemple) sont exprimées indirectement au travers de l'évitement et de l'absence de réponse. Vu la forte tendance, à base évolutionniste, des bébés humains à rechercher le contact quand ils souffrent, l'échec à le faire reflète une distorsion majeure de la régulation émotionnelle et compromet la capacité du bébé à retourner à une exploration active de qualité (Ainsworth, et al., 1978).

L'attachement insécure ambivalent ou résistant se caractérise par une inefficacité à obtenir la sécurité de la part de la figure d'attachement, malgré des efforts répétés pour la solliciter. Les bébés avec des histoires d'attachement résistant peuvent se sentir méfiants face à l'étranger et montrer une exploration pauvre, en pleurant fréquemment ou en cherchant le contact avec la figure d'attachement même avant la séparation. La simple présence du caregiver ne paraît pas les tranquilliser. Les séparations sont extrêmement stressantes et les bébés peuvent confondre la recherche de contact avec l'agressivité, la tension corporelle et les pleurs persistants lors de la réunion avec la figure d'attachement. L'anxiété et la colère exprimées interfèrent avec les tentatives du bébé pour obtenir du réconfort grâce à la proximité, et les empêchent d'explorer leur environnement. Le manque de confiance vis-à-vis de la figure d'attachement est évident.

Le groupe de bébés avec un attachement désorganisé/désorienté, un pattern plus récemment découvert, met en évidence une série de réponses comportementales indirectes ou mal dirigées, dans le contexte de la Situation Etrange.

Main et Salomon (1990) ont été les pionniers de l'identification des signaux de désorganisation de l'attachement chez les bébés de 12-18 mois, observés en présence de la figure d'attachement: a) manifestation séquentielle et/ou contradictoire de comportements; b) mouvements et expressions non dirigés, incomplets ou interrompus; c) stéréotypies, mouvements asymétriques et postures anormales; d) mouvements et expressions de stupéfaction, immobilisation et lenteur; et e) expressions d'appréhension et de peur relativement à la figure d'attachement ${ }^{11}$. 
La désorganisation de l'attachement a été associée à des comportements parentaux très perturbés et terrifiants (frightened/frightening) pour les enfants qui vivent une situation de stress. Dans ces conditions, la figure parentale peut générer la peur et l'appréhension, en mettant l'enfant dans une situation de conflit insoluble, puisque la source d'apaisement est aussi la source de la peur, et que l'enfant ne peut simultanément rechercher et fuir sa figure d'attachement ${ }^{12}$ (Main et Hesse, 1990).

L'évaluation en âge préscolaire se doit de porter une attention particulière aux nouveaux défis de développement:1) le système d'attachement n'est pas, maintenant, aussi facilement activé; 2) le répertoire comportemental exhibé par les enfants est plus riche; et 3) ses capacités linguistiques et représentationnelles (pour une possible évaluation au travers du discours) sont encore primitives et très variées. Les évaluations disponibles incluent le Marvin et Cassidy Attachment Classification System (Cassidy et Marvin, 1992), le Preschool Assessment of Attachment (Crittenden, 1994), et le Main and Cassidy Classification System (Main et Cassidy, 1988).

Les études transculturelles plaident en faveur de l'universalité de l'attachement (Bowlby, 1969/1982) et de la présence des principaux patterns d'attachement dans l'enfance dans diverses cultures et contextes sociaux (Van Ijzendoorn et Sagi, 1999). Dans les différentes cultures, et indépendamment du style de caregiving (p. ex.: famille nucléaire ou réseau de prestation de soins), sauf en cas d'extrême détérioration neurologique ou de grave privation de soins, les bébés ont tendance à organiser leurs comportements d'attachement autour de prestations de soins spécifiques $^{13}$.

Les trois patterns de base de l'attachement (c.-à-d.: attachement sécure, évitant et résistant) ont été observés au sein de cultures occidentales et non occidentales, en Afrique, en Chine, en Israël, au Japon, en Europe (Angleterre, Allemagne, Suède, Espagne, Portugal ${ }^{14}$ ) ainsi qu'en Amérique du Nord et en Amérique du Sud (Canada et Etats-Unis, Mexique et Colombie). Dans ces cultures, la majorité des enfants présentent des relations d'attachement sécure. Des variations culturelles spécifiques ont encore été observées pour les distributions insécures et leurs sous-groupes. Ayant pour base d'investigation l'AQS (Vaughn et Waters, 1990), les experts et les mères paraissent conceptualiser et évaluer la sécurité de l'attachement d'une façon semblable dans diverses cultures (Colombie, Allemagne, Israël, Japon et Etats-Unis) (Posada, Waters, Crowell, et al., 1995). 


\section{Qualité des relations d'attachement}

Selon la théorie de l'attachement, la nécessité de contact humain, de tranquillisation et de réconfort face aux maladies, aux dommages physiques et aux menaces est une réponse normale tout au long du cycle de vie. Cette nécessité est particulièrement apparente dans les phases initiales du développement, quand la survie physique et émotionnelle dépend de la relation d'attachement.

L'hypothèse de Bowlby, mentionnée antérieurement, qui avance que les différences dans le caregiving donnent lieu à des différences dans la qualité de l'attachement, a reçu un support empirique. La réponse du caregiver pendant la première année de la vie se trouve associée à la qualité de la relation d'attachement évaluée autant dans un contexte familier qu'en laboratoire: une réponse adaptée et efficace aux signaux du bébé est associée à un attachement sécure dans la Situation Etrange (à 12 mois) et à moins de comportements mal-adaptatifs (p. ex.: pleurs excessifs et exploration pauvre; Ainsworth, et al., 1978).

Au contraire, un soin moins sensible dans le contexte familier (c.-à-d.: retard dans les réponses à la perturbation du bébé et comportements moins affectueux) a été mis en relation avec un attachement insécure évalué en laboratoire (Ainsworth, et al., 1978 ; Blehar, Lieberman, et Ainsworth, 1977). Le caregiving caractérisé par l'indisponibilité émotionnelle et le rejet chronique est en lien avec une relation insécure évitante (Main, 1981). Les soins simultanément intrusifs et peu adaptés, peu congruents et peu fiables, ont été associés à un attachement insécure résistant.

D'après les études d'Ainsworth et al. (1978), l'attachement sécure est relié aux comportements maternels suivants: a) contact physique fréquent et soutenu entre le bébé et sa mère, spécialement pendant les six premiers mois, capacité maternelle à calmer son bébé, en le prenant dans ses bras; b) sensibilité maternelle aux signaux du bébé, et, en particulier, capacité à gérer ses interventions en harmonie avec les rythmes du bébé; c) une ambiance contrôlée et prévisible, qui permet au bébé d'inférer les conséquences de ses propres actions; d) plaisir mutuel ressenti par la mère et le bébé.

L'évitement est associé à quatre aspects principaux du comportement maternel: a) rejet chronique; b) rejet spécialement communiqué par des réactions au contact corporel proche; c) colère contenue; et d) ajustement généralement compulsif. Main (1977) a avancé l'hypothèse que le relatif manque d'expressivité émotionnelle, caractéristique des mères du groupe évitant, peut être attribué à un effort des mères pour contrôler 
leur colère. C'est le rejet implicite de la colère ressentie qui affecte le bébé, malgré la tentative maternelle de la contenir. On pense qu'un ajustement compulsif de ce type rend la mère moins consciente des signaux du bébé et, par conséquent, moins à même de répondre efficacement (Ainsworth, et al., 1978).

La relation entre sécurité dans l'attachement en laboratoire et sensibilité du caregiver en contexte familial a été amplement confirmée (Grossmann, Grossmann, Spangler, et al., 1985; NICHD, 1997), spécialement par les investigateurs proches de la méthodologie utilisée par Ainsworth (c.-à-d.: observations extensives, mesures adaptées et bébés d'âges comparables) (Pederson, Gleason, Moran, et Bento, 1998; Posada, Jacobs, Carbonell, et al., 1999).

Des études récentes ont aussi confirmé le lien entre le pattern d'attachement désorganisé et une série de comportements parentaux effrayants ou confus (Jacobitz, Hazen, et Riggs, 1997; Lyons-Ruth, Bronfman, et Parsons, 1999). La relation entre l'expérience de prestation de soins et la qualité de l'attachement est encore attestée par des études en situation de mauvais traitements. Les bébés et petits enfants objets de mauvais traitements ont une tendance significativement plus élevée à former des relations d'attachement insécure avec leurs figures d'attachement primaire (c.-à-d.: évitant, résistant et désorganisé), bien plus que les bébés de familles au statut socio-économique bas (Carlson, et al., 1989; Egeland et Sroufe, 1981; Carlson, 1998; Crittenden, 1988; Lyons-Ruth, Repacholi, McLeod, et al., 1991; Schneider-Rosen, Braunwald, Carlson, et Cichetti, 1985). La désorganisation de l'attachement des bébés a aussi été mise en relation avec des expérience parentales de pertes, de facteurs psychosociaux (p. ex.: dépression) (Lyons-Ruth, et al., 1991; Radke-Yarrow, Cummings, Kucznyski, et al., 1985), d'ambiance de soins associées à l'alcoolisme prénatal (O'Connor, Sigman et Brill, 1987) et d'exposition aux drogues (Rodning, Beckwith, et Howard, 1991).

La contribution du tempérament du bébé à la sécurité de l'attachement a été amplement étudiée: bien qu'aucune liaison directe n'ait été trouvée entre le tempérament initial du bébé et la qualité de l'attachement (Vaughn et Bost, 1999), le tempérament est associé à certains aspects de l'attachement. La propension à la perturbation et à la réactivité au cortisol prédit l'intensité des pleurs durant les séparations dans la Situation Etrange à 9 mois (Gunnar, Mangelsdorf, Larson, et Hertsgaard, 1989; Spangler, et Grossmann, 1993). Toutefois, ce niveau de perturbation ne prédit pas la qualité de l'organisation de l'attachement ou la facilité 
d'obtenir du réconfort dans la présence de la figure d'attachement - le grand marqueur de sécurité.

Comme la sécurité vis-à-vis de la figure d'attachement est en relation avec l'histoire interactive de la dyade, le même enfant peut développer un attachement sécure avec un des parents et un attachement insécure avec l'autre (Grossmann, Grossmann, Huber, et al., 1981; Main et Weston, 1981), ou passer d'un attachement insécure vers un attachement sécure avec le même parent quand surviennent des changements significatifs dans les circonstances de vie (Egeland et Farber, 1984).

Il est à souligner que certains bébés peuvent se montrer très difficiles et mettre en péril le système de caregiving . Toutefois, quand un soutien aux parents est donné de façon adéquate, les soins sensibles permettent de mieux affronter les difficultés de ces enfants (Crokenberg, 1981; Susman-Stillman, Kalkowske, et Egeland, 1996). Le risque d'un attachement insécure semble provenir d'une combinaison spécifique des exigences élevées du bébé associées à des difficultés inhérentes au caregiver (p. ex.: propension du bébé à la perturbation associée à un type de soins exagérément rigides; Gunnar, 2001; Mangeldorf, Gunnar, Kestenbaum, et al., 1990).

Les changements dans le caregiving varient depuis des changements de routine (p. ex.: soins en crèches) jusqu'aux changements très graves (p. ex. : multiples colocations en familles d'accueil temporaire et institutionnalisation), avec des conséquences très diverses. En général, les effets dépendent du moment, de la durée de la perturbation et de la mesure dans laquelle la disponibilité émotionnelle de la figure d'attachement est compromise.

Les conséquences de la vie en crèche pour la sécurité de l'attachement ont aussi été étudiées: en général, quand le séjour en crèche pendant la première année de la vie est à temps partiel, qu'il est stable et de qualité élevée, on ne met en évidence aucune conséquence négative (Sroufe et Ward, 1984). Toutefois, les soins précoces en crèche à temps complet peuvent présenter des risques, étant donné que les résultats négatifs les plus évidents surviennent en la présence simultanée de soins de qualité réduite à la maison et hors de la maison (Belsky, 1990; NICHD, 1997, 2007). La recherche a souligné l'importance de la qualité de soins hors du contexte familial pour le développement postérieur des enfants (Anderson, 1992; Vandel et Corasaniti, 1990).

Les perturbations plus graves de caregiving dans l'enfance incluent les hospitalisations, les séparations, les expériences d'adoptions et de 
familles d'accueil temporaires. Robertson et Robertson, en collaboration avec Bowlby, ont été les premiers à documenter les effets sur le bien-être de l'enfant des conséquences de la séparation enfant-figure d'attachement au cours des hospitalisations précoces (Robertson, 1953; Robertson et Robertson, 1971). L'évidence de signaux de deuil chez les bébés (c.-à-d.: protestation, désespoir et détachement) après des séparations prolongées a alerté les professionnels de santé et généré des changements significatifs dans les pratiques et politiques pédiatriques de plusieurs pays. Malgré ces découvertes et les liens découverts entre expériences d'hospitalisation précoce et perturbations postérieures du comportement (Douglas, 1975; Quinton et Rutter, 1976), peu d'études ont examiné l'impact de l'hospitalisation dans le développement des relations d'attachement.

La sécurité dans la relation d'attachement en cas d'adoption a été associée au moment de la transition et à la qualité des soins. Les études réalisées dans ce contexte suggèrent que les bébés adoptés lors des premiers mois de la vie ont une probabilité élevée de développer des relations d'attachement sécures presque équivalentes aux bébés non adoptés (Norhaus et Solnit, 1990; Singer, Brodzinsky, Ramsay, Steir, et Waters, 1990). En particulier, quand l'ambiance est chaleureuse, consistante et contingente vis-à-vis des besoins du bébé, l'attachement en situation d'adoption, lorsqu'il est construit tôt dans le processus de développement, diffère peu de celui qui est établi entre parents et leurs enfants biologiques (Singer et al., 1985). Toutefois, les enfants adoptés à des âges plus tardifs ont une plus grande probabilité de présenter des difficultés d'ajustements socio-émotionnels et comportementaux (Feigelman et Silverman, 1983; Tizard, 1977; Tizard et Hodges, 1978). Ces difficultés dans les adoptions tardives sont multi-déterminées et incluent les expériences antérieures à l'adoption et d'autres facteurs associés au moment de l'adoption (c.-à-d.: expérience de l'enfant et compréhension de la séparation et de la perte).

Actuellement, on a commencé à étudier les effets du caregiving en familles d'accueil temporaire sur le développement de l'organisation de l'attachement. Les découvertes initiales confirment les effets sévères des expériences précoces de mauvais traitements (p. ex.: abus et négligence émotionnelle et physique), séparation, perte et placements multiples (Stovall et Dozier, 2000; Fisher, Gunnar, Chamberlain, et al., 2000). Les perturbations traumatiques dans le caregiving paraissent amener des distorsions dans les relations d'attachement et dans le développe- 
ment de comportements mal-adaptatifs (p. ex.: sociabilité indiscriminée et terreur des étrangers) et des perturbations dans la perception et l'interprétation des indices sociaux (Stovall et Dozier, 2000).

La grande importance de la communication affective entre les bébés et leurs parents est illustrée autant par des recherches classiques que par des recherches relatives aux effets de l'institutionnalisation dans le développement des enfants. Les études classiques de Spitz (1946) ont mis en évidence que le développement perceptif, moteur, cognitif et émotionnel du bébé survient au travers de l'interaction avec l'adulte, figure d'attachement primaire, et l'absence d'une relation avec un partenaire humain peut être le moteur d'une désintégration du développement. La privation, suivie d'une mise en famille d'accueil temporaire, a été associée à des problèmes émotionnels et comportementaux (p. ex. : comportements hétéro- et auto-agressifs, recherche d'attention, hyperactivité et troubles alimentaires). En accord avec les études initiales, les recherches récentes avec des enfants en institution (Chisholm, 1998; Kaler et Freeman, 1994; Marcovitch, Goldberg, Gold, et al., 1997; O'Connor, Bredenkamp, Rutter, et al., 1999) ont révélé des difficultés relationnelles majeures dans leurs familles adoptives (p. ex.: agression, sociabilité indiscriminée, hyperactivité et problèmes avec les partenaires). Ces effets sont plus nets quand l'institutionnalisation perdure au-delà de la première année de vie, en comparaison avec ceux d'une institutionnalisation limitée à quelques mois (Gunnar, 2001; O’Connor, et al., 1999).

\section{Attachement et psychopathologie}

Les perturbations de la relation d'attachement ne sont pas considérées comme des perturbations propres à l'enfant, mais comme des marqueurs relationnels d'un processus pathologique, et comme un facteur de risque pour la survenue d'une pathologie subséquente (Sroufe, Carlson, Levy, et Egeland, 1999; Sroufe, Duggal, Weinfield, et Carlson, 2000)15.

Un attachement sécure ne garantit pas le bien-être, mais il augmente la résistance au stress et promeut la résilience (Pianta, Egeland et Sroufe, 1990). Les patterns d'attachement insécure dans l'enfance sont des adaptations provisoires qui maximisent, dans la mesure du possible, le maintien de la proximité avec la figure d'attachement dans un contexte d'indisponibilité ou de disponibilité intermittente de cet adulte. Les enfants aux patterns insécures évitants maintiennent la proximité au caregiver en minimisant les signaux de stress et de négativité qui peuvent l'aliéner et 
susciter le rejet (Main, 1981). Pour les enfants classés comme insécures ambivalents ou résistants, l'augmentation de l'intensité des signaux de stress maintient activement l'attention de figures d'attachement dont les réponses sont intermittentes. Même la désorganisation de l'attachement (p. ex.: stéréotypes, comportements simultanés d'approche/évitement) peut permettre à l'enfant de maintenir la proximité dans un contexte de comportements parentaux effrayants et de conflit interne (Main et Hesse, 1990). Ces patterns d'attachement mettent en jeu le développement à long terme, en limitant la capacité d'adaptation de l'enfant. Les individus avec des histoires insécures sont ainsi potentiellement plus susceptibles de former des relations affectives de non-support et facilement perturbées, et perdent ainsi des recours importants contre le stress et la psychopathologie. Pour les individus dont l'histoire personnelle est extrêmement sévère, chaotique et traumatique (désorganisation de l'attachement), l'intégration d'expériences comportementales et émotionnelles est compromise, et les pensées et images intrusives ont tendance à intervenir de façon imprévisible et à générer des perturbations dans les relations d'attachement (Carlson et Sroufe, 1995).

Les modes dysfonctionnels de régulation précoce prédisposent les individus à des types spécifiques de comportements symptomatiques et de perturbations (Bowlby, 1988; 1973/1979; 1979; 1980). Pour des individus avec des histoires d'attachement insécure de type évitant, le comportement symptomatique parait lié à une tentative de minimiser leurs comportements d'attachement et leurs sentiments (dissimulation de l'expression émotionnelle, et croyances relatives à un soi vécu comme invulnérable, à l'idée que les autres sont hostiles et non dignes de confiance, à des relations idéalisées). L'aliénation, le manque d'empathie et l'hostilité peuvent prédisposer ces individus à des troubles de conduites et des styles de personnalité antisociale.

Les individus aux histoires insécures résistantes, avec la baisse tolérance à la frustration, la tendance à exagérer les émotions et au maintien de croyances négatives relatives au self, peuvent être amenés, à long terme, à des sentiments de confusion face aux défis posés par l'exploration et à des difficultés accrues dans la gestion de l'anxiété (Bowlby, 1973/1979 ; Kobak et Shaver, 1987).

Les deux patterns insécures peuvent contribuer à augmenter la vulnérabilité face à la dépression (c.-à-d.: aliénation et solitude; passivité, désespoir et impotence) et au développement de perturbations face à la séparation et à la perte - expériences qui ont tendance à confirmer les 
expectatives sur l'indisponibilité psychologique des figures d'attachement significatives (Bowlby, 1980). Les individus avec un attachement désorganisé, caractérisé par une impossibilité de maintenir une stratégie d'attachement cohérente et par des postures ressemblant à des états de transe (Main et Hesse, 1990), sont en risque majeur de psychopathologie de diverses natures, incluant des perturbations de type dissociatif (Carlson, 1998; Lyons-Ruth, et al., 1991; Main et Morgan, 1996).

Les données longitudinales (c.-à-d.: études en grande section de l'école maternelle, à l'école élémentaire et à l'adolescence) confirment les associations entre l'expérience relationnelle d'attachement et les indicateurs généraux de mauvaises adaptations. Les attachements insécures mettent les enfants à risque de développer des problèmes comportementaux, quand ils sont combinés avec des situations de vie stressantes (décès d'un proche, divorce, changement de domicile de la famille,...) et avec des variations familiales (par exemple, naissances) (Erickson, Egeland, et Sroufe, 1985; Renken, Egeland, Marvinney et Sroufe, 1989). Cela est manifeste quand le caregiving et les facteurs contextuels négatifs sont permanents. Les changements de ces trajectoires attachementales ont été associés à des changements dans les circonstances contextuelles (p. ex.: soutien social). Des mesures composites de l'expérience précoce, incluant l'attachement, prédisent elles aussi la capacité d'adaptation des enfants en âge scolaire et des adolescents (p. ex. : classifications de santé émotionnelle attribuées par les professeurs; Sroufe, et al., 1999; Sroufe, Egeland, et Kreutzer, 1990). Dans le cas particulier de la désorganisation de l'attachement des enfants, cette expérience précoce prédit significativement la psychopathologie de l'adolescence, surtout si on considère l'existence de problèmes comportementaux au long de l'enfance et la qualité de la relation caregiver - enfant (Carlson, 1998).

Dans l'étude longitudinale du Minnesota, des associations spécifiques ont été trouvées entre l'expérience précoce d'attachement et le comportement antisocial, l'anxiété, la dépression et la symptomatologie dissociative (Collins, 2005). Des histoires initiales d'attachement évitant ont pu être associées à l'agressivité dans la deuxième enfance (Renken et al., 1989) et à l'agressivité et la délinquance dans l'adolescence, spécialement chez les garçons. Le rôle de l'histoire précoce se reflète aussi dans l'étude de deux types de comportement antisocial des adolescents (Aguilar, Sroufe, Egeland, et al., 2000). Les sujets qui appartiennent à des groupes de pairs manifestant des comportements antisociaux (à partir de l'enfance ou de l'adolescence, et persistant tout au long de la vie) se distinguent 
par leur histoire socio-émotionnelle pendant leurs trois premières années de vie - monoparentalité, dépression maternelle, stress de vie, sensibilité maternelle réduite, type d'attachement insécure, mauvais traitements et peu de soutien maternel - mais pas par leur tempérament. L'attachement évitant, en combinaison avec d'autres événements de vie stressants et d'autres facteurs de risques, peut promouvoir le développement de comportements anti-sociaux (Greenberg, Speltz, DeKlyen, et al., 1991).

La perturbation face à la séparation peut cependant rester adaptative, dans un contexte de soins sensibles (c.-à-d.: signalisation rapide du bébé et comportement de recherche et de proximité); cependant, dans une ambiance de soins inconsistants, la vigilance chronique et l'anxiété peuvent contribuer à établir un mode de réponse qui se généralisera à de multiples sources de peurs et au développement d'un trouble anxieux.

Les données longitudinales de l'étude du Minnesota ont encore déterminé que les facteurs psychosociaux précoces contribuent au développement de la symptomatologie dépressive, autant à l'enfance qu'à l'adolescence (Duggal, Carlson, Sroufe, et al., 2001). Le manque de soutien émotionnel envers l'enfant et son lien avec la dépression ont apporté une immense contribution à la compréhension de la dépression dans l'adolescence. A partir de la variable composite petite enfance, l'attachement insécure prédit significativement la symptomatologie à l'adolescence, même en contrôlant tous les autres facteurs. Ces découvertes indiquent que les expériences de dérégulation émotionnelle dans la petite enfance peuvent avoir un effet durable sur l'expérience dépressive adolescente, étant donné que celle-ci se trouve associée au même phénomène chez l'adulte.

L'attachement désorganisé et les antécédents de trauma ont été liés sur le plan théorique à l'apparition de symptômes de type dissociatifs, autant à l'enfance qu'à l'adolescence (Main et Hesse, 1990; Putnam, 1994; Liotti, 1992; 2005). En réponse à un conflit précoce ou à une menace, les bébés ayant une histoire de désorganisation excluent certains aspects de l'expérience affective, empêchant le développement cohérent d'une organisation du self et des autres dans la relation. Dans une situation de trauma, ces enfants ont tendance à répondre par des modèles de déconnexion de stimuli préalablement établis, ce qui est un élément perturbateur pour un traitement cognitif et émotionnel normal (Liotti, 1992). Les données longitudinales confirment les relations entre désorganisation et attachement, trauma et symptomatologie dissociative (Carlson, 1998; Collins, 2005; Ogawa, Sroufe, Weinfield, et al., 1997). L'attache- 
ment désorganisé a été associé à la dissociation dans l'enfance et adolescence, avec des évaluations cliniques à 17 ans et demi (Kiddie Schedule for Affective Disorders and Schizophrenia) et des auto-questionnaires à 19 ans (Dissociative Experiences Scale). Le traumatisme, à chacun de ces âges, a été mis en rapport avec la dissociation actuelle et avec la symptomatologie de la prochaine période, spécialement dans les cas de trauma chronique. Ces données suggèrent que la chronicité du traumatisme peut augmenter la probabilité de dissociation. De plus, certains individus peuvent être spécialement vulnérables à des modèles dissociatifs face au trauma parce que les comportements proto-dissociatifs précoces peuvent avoir été établis parmi des relations de soins.

Il est toutefois indispensable de souligner qu'en général, les études longitudinales indiquent que la majorité des différences d'attachement chez les bébés ne sont pas pathologiques. Au contraire, les variations dans les patterns d'attachement représentent des conditions initiales qui accomplissent un rôle dynamique dans le développement de la pathologie et de la résilience. Les effets durables des expériences précoces peuvent dériver de la forme - de l'ambiance qui appuie ces tendances et expectatives initiales -, et de l'impact de l'expérience précoce dans la régulation neurophysiologique et affective de base. Autant la théorie que l'investigation démontre que le changement, bien que plus facile dans le jeune âge, reste possible au long du développement.

Dans cette ligne d'idée, la sécurité dans l'attachement ne signifie pas l'immunité face aux souffrances psychologiques, mais peut aider au développement de compétences qui les minimisent ou qui aident à les vaincre. S'il existe une histoire de vie dans laquelle l'individu a eu des occasions d'interagir avec des figures d'attachement qui ont fonctionné comme havre de sécurité et base de sécurité (et, à travers elles, de ressentir le «self» comme compétent et méritant l'attention et le soutien des autres), en situations d'adversité le recours à des relations d'aide sera plus facile.

Bretherton (1990) suggère que la capacité de l'individu pour discuter, de manière ouverte et cohérente, des questions d'attachement, dépend de l'adaptation de ses modèles internes dynamiques du self et des autres dans les relations d'attachement. La façon de répondre des parents aux communications de leur enfant peut modifier leurs modèles d'interaction et les modèles internes opérants des deux partenaires (Tereno, 2004). L'absence d'une communication ouverte entre les partenaires peut être associée à une restriction cognitive de la fluidité de l'information sur l'attachement. Ceci s'applique, non seulement entre les deux sujets, 
mais aussi dans le système représentationnel de chacun, car le matériel défensivement exclu ne peut pas participer aux processus de correction d'erreurs par feedback du système mnésique de l'individu (Bretherton, 1990; Jang, Smith, et Levine, 2002; Dallos, 2004).

On sait encore qu'une histoire de vie marquée par la confiance dans les relations (c.-à-d.: la communication directe des émotions, la flexibilité et l'ouverture dans l'interprétation de l'information, et la capacité de réfléchir aux états du «self» et des autres) pourra constituer, en situation difficile, une base de sécurité pour un travail psychologique réussi, ainsi que pour la bonne résolution des expériences de perte ou de trauma. L'investigation empirique a mis en évidence que, dans le processus psychothérapique, les familles avec une organisation sécure font preuve d'une plus grande capacité d'ouverture et sont perçues par les thérapeutes comme plus coopérantes dans les tâches et les objectifs de la thérapie, comparées à d'autres familles avec une organisation insécure. De plus, l'organisation de l'attachement sécure paraît être associée à des résultats plus favorables en psychothérapie (Dollan, Arnhoff, et Glass, 1993; Dozier, 1990; Dozier, Cue, et Barnett, 1994; Korfmacher, Adam, Ogawa, et Egeland, 1997; Lieberman, Weston, et Pawl, 1991).

\section{Implications cliniques}

La théorie et l'investigation de l'attachement fournissent une structure utile à l'intervention clinique, ainsi qu'un guide des pratiques cliniques et des soins parentaux généraux (Berger, 1994). La pratique pédiatrique accompagne de nombreuses dimensions du développement de l'enfance précoce et de la dynamique relationnelle parent-enfant. Fréquemment, les pédiatres sont les premiers à observer et à comprendre les perturbations des enfants, chez le parent ou dans la relation dyadique. Toutefois, malgré l'attention prêtée aux perturbations d'interaction caregiver-enfant, il est difficile de diagnostiquer les difficultés d'attachement spécifiques lors de consultations cliniques de brève durée. Les comportements qui peuvent être critiques s'ils sont chroniques, peuvent être complètement appropriés face à une menace ou à des situations spécifiques. Au-delà, il est clair que toutes les difficultés relationnelles parent-enfant n'impliquent pas la présence d'une perturbation de l'attachement (Sameroff et Emde, 1989).

Pour identifier les forces et les vulnérabilités relationnelles et pour une bonne gestion clinique du cas, des évaluations sont nécessaires, qui 
incluent l'histoire psychosociale, des observations comportementales directes, un entretien avec les parents et, quand cela est approprié, le recueil d'auto-questionnaires de l'enfant. Cette investigation devra intégrer l'exploration de questions importantes comme les expériences de séparations, de perte, d'abus, et des signaux de comportements parentaux inadaptés ou atypiques. Les marqueurs observationnels peuvent inclure l'absence ou la distorsion du comportement de base de sécurité (c.-à-d.: présence de monitorage visuel ou physique, mais peu fréquent), incapacité de s'engager dans l'exploration (c.-à-d.: extrême inhibition, peur ou dépendance), perturbation extrême ou absence de perturbation lors d'une séparation avec le caregiver, agressivité extrême ou difficulté à établir des interactions avec le caregiver, contrastant avec le comportement de familiarité excessive avec les étrangers.

De même que les difficultés relationnelles varient entre des perturbations temporaires et celles qui sont à risque élevé pour le développement futur de perturbations graves, les options d'intervention peuvent varier du soutien relationnel à l'intervention psychothérapique dyadique intensive (Lojkasek, Cohen, et Muir, 1994). Le traitement des perturbations graves associées à l'attachement est complexe. Pour les enfants qui ne montrent pas de préférence à l'égard d'une figure d'attachement, le plus important dans l'intervention est le fait de promouvoir un contexte relationnel où l'enfant ait, à la fois, la disponibilité et la sensibilité des parents - un contexte dans lequel l'attachement peut se développer (Robinson, 2002). Pour les enfants qui ont subi une privation relationnelle précoce, des séparations multiples ou durables, ou des traumas précoces, le processus d'intervention est généralement long et le changement peut être irrégulier, posant ainsi des défis de taille aux parents. L'évolution de l'attachement et de la régulation de l'émotion, particulièrement après l'enfance, n'est pas nécessairement parallèle au développement attendu. Par exemple, les comportements d'attachement peuvent s'exprimer sous forme d'une perturbation excessive dans la séparation ou inconsolable lors des rencontres, de crises de colère ou de rage face à la moindre frustration, ou de comportements d'attachement très intenses et exigeants. Tandis que la relation d'attachement s'établit, les comportements mal adaptés (par exemple, sociabilité indiscriminée et comportements à risque) peuvent persister. Les stratégies d'interventions intégratives dirigées vers les questions de développement multiple et vers l'éducation et le soutien parental sont donc fondamentales. 


\section{Résumé}

Les pédiatres sont fréquemment les premiers professionnels à observer les relations de soins précoces et leurs impacts dans le développement de l'enfant. Les relations précoces de caregiving, leur formation, leur qualité et les questions de développement associées se trouvent amplement inclues dans le cadre de l'évaluation pédiatrique. Cet article présente une vision générale de la théorie et la recherche de l'attachement qui pourra être utile comme grille de lecture de la qualité des soins et de l'organisation de la relation du bébé avec ses figures parentales. Nous décrirons les phases de développement des relations d'attache-
Pour les enfants et les parents dont les relations sont déjà établies, les variations au sein du modèle thérapeutique peuvent représenter des différences dans les portes d'accès directs et indirects au système dyadique (comportement de l'enfant; représentation et comportements parentaux; interaction enfant-caregiver; soutien à la relation) (Stern, 1995). Ces variations de techniques psychothérapeutiques enfant-parent ne sauraient être évaluées précisément en termes de résultats. Des effets différentiels, spécialement en ce qui concerne les différentes sous-populations, dérivent de la nature du cadre thérapeutique et de la relation avec le thérapeute. Les aspects importants du traitement, communs aux différentes approches, incluent l'aspect séquentiel (comme la méthode "Touchpoints» de Brazelton, 1992) qui caractérise le développement rapide de l'organisme de l'enfant et du système familial, l'importance d'une relation positive dans la thérapie, et une emphase sur les forces relationnelles et sur l'expérience émotionnelle. D'une façon générale, la revue de la littérature indique qu'il n'existe pas de solutions rapides pour les perturbations relationnelles parent-enfant. Les stratégies d'intervention sont importantes, incluant les protocoles déjà établis, qui enseignent les pratiques de parentalité, via la construction de relations de travail et de partenariat avec les parents basées sur la sensibilité et l'empathie (Robinson, 2002).

En général, la recherche sur l'intervention indique que, spécialement chez les populations à risque élevé, l'intervention doit commencer tôt, inclure directement les parents, se rapporter aux aspects émotionnels de la relation de soins et, plus important encore, garantir une ambiance thérapeutique sécure et soutenante (Robinson, 2002; Egeland, Weinfield, Bosquet, et al., 2000).

Le travail clinique est naturellement riche en transmission générationnelle; les histoires que nous étudions émergent de scénarios interpersonnels qui s'étendent tout au long du développement des protagonistes. Ce travail nous amène à croire à la capacité de transformation personnelle et interpersonnelle: les histoires de vie marquées par l'adversité ne produisent pas toujours d'autres histoires négatives. Le cycle peut se rompre: même sans connaître en profondeur les mécanismes de transmission et de changement, nous savons que le changement vers la sécurité peut survenir grâce à l'engagement dans une relation émotionnellement investie et féconde. La psychothérapie peut être une opportunité pour explorer de nouveaux parcours et pour construire de nouveaux sens à l'existence personnelle et familiale (Soares, 2002). 
Au-delà du fait de donner des lignes directives à la psychothérapie, la théorie et l'évaluation de l'attachement ont constitué la base de modèles psychothérapeutiques. Dans ce contexte, on peut distinguer le modèle constructiviste de développement de Guidano $(1987,1991)$ et le modèle cognitiviste évolutionniste de Liotti (2000). Dans le contexte psychanalytique, quelques auteurs ont cherché à systématiser les applications à la psychothérapie de la théorie et de l'investigation de l'attachement, tels Holmes (2001), Fonagy (2001) et Slade (1999). On distingue encore les contributions de Byng-Hall $(1999$; 2001) dans une perspective systémique, les applications en thérapie conjugale proposée par Clulow (2001) et l'approche psychothérapeutique parents-enfant, proposée par Lieberman, Weston et Pawl (1991), toutes particulièrement importantes pour la thématique pédiatrique. Reflétant l'intérêt croissant pour les applications cliniques, un numéro du magazine Attachment and Human Development a été précisément consacré à cette problématique dans le contexte des institutions de santé mentale (cf. Schuengel et van IJzendoorn, 2001).

\section{Conclusion}

Une des tâches primordiales de l'enfance est la formation de relations d'attachement envers les figures significatives prodiguant les soins. La théorie et les études empiriques de l'attachement indiquent que les variations dans la qualité des soins donnent lieu à différents patterns comportementaux et de régulation émotionnelle chez l'enfant, et à des attentes qui ont des effets dans le fonctionnement individuel à long terme. Ces prémisses centrales de la théorie de l'attachement nous rappellent la nécessité humaine du contact physique et de la garantie émotionnelle dans l'enfance. Si l'on considère l'universalité de ce besoin et ses implications pour la survie, il n'est pas surprenant que les enfants s'attachent à ceux qui les soignent. Toutefois, comme les différences individuelles observées dans l'attachement sont décrites par des variations de modélisation du comportement, les modèles de description quantitative communément utilisés (comme attaché, pas attaché, très ou peu attaché) ont peu de sens, n'étant pas utiles pour expliquer les relations précoces.

Les relations d'attachement se développent par phases: à partir des relations non discriminantes (figures d'attachement interchangeables), à l'organisation de l'attachement autour d'une personne préférée (caregiver qui répond avec le plus de fréquence aux nécessités du bébé), à l'organisation comportementale autour d'une hiérarchie de figures d'attachement.

ment, et la manière d'évaluer les facteurs influençant les variations dans l'organisation de l'attachement. Dans une perspective développementale, la relation entre attachement et psychopathologie sera aussi explorée. Nous finirons par une brève revue des implications cliniques de la théorie de l'attachement pour l'exercice de la pédiatrie.

\section{Mots clés}

Attachement.

Développement.

Pédiatrie. 


\section{Summary}

Pediatricians are frequently the first professionals to observe the early caregiving relationships and their impact in the development of the child. These early relationships, their formation, quality and related issues on development are amply included within the pediatric assessment framework. This paper presents a general vision of attachment theory and research, which can be useful to understand the quality of the care as well as the baby's attachment organisation to his parental figures. We will describe the
La théorie et l'investigation de l'attachement indiquent qu'il n'existe pas de période critique unique après la naissance ou à un âge précoce qui consolide la relation figure d'attachement-enfant ${ }^{16}$. Le contact peau à peau ou psychologique de la mère avec l'enfant après la naissance n'est pas un prérequis pour l'attachement, bien qu'il marque le début de certains processus parentaux importants. Les attachements des bébés aux figures d'attachement primaires, ainsi que les liens de ces derniers aux premiers, se construisent au fil du temps au travers de multiples interactions. C'est une disponibilité physique et émotionnelle consistante qui s'avère critique pour le développement d'attachement sécure pour les bébés et qui fonde le fonctionnement adaptatif.

Les relations d'attachement sont spécifiques à chaque personne. Les bébés construisent des patterns de relations d'attachement différents avec des donneurs de soins distincts, et la qualité de chaque relation dépend de la qualité de l'interaction et du soin. L'investigation des effets d'une ambiance construite par de multiples caregivers souligne l'importance du temps individualisé pour chaque dyade parent-enfant ${ }^{17}$, ainsi que le support social et émotionnel fourni à la relation. Ce soutien socioémotionnel a un impact sur les relations d'attachement à divers niveaux: a) il facilite l'expression de la sensibilité des parents, sans les surcharger; b) il fournit des relations alternatives aux enfants à risque; c) il modère les effets du tempérament des enfants difficiles; et d) il assiste les relations caractérisées par des besoins atypiques (tels qu'enfants handicapés ou avec des retards de développement).

Contrairement aux croyances populaires, la réponse aux pleurs d'un bébé de moins de douze mois n'augmente pas sa dépendance à l'égard des adultes. En effet, les bébés auxquels, dans les premiers trois mois, on répond toujours et aisément, pleurent moins à la fin de la première année (Ainsworth et Bell, 1974). Les réponses sensibles et consistantes aux signaux de l'enfant leur donnent le retour de leur comportement de signalisation, inculquant au bébé un ensemble d'attentes en rapport à leurs compétences et à leur valeur propre. De la même manière, un soin consistant et une réponse empathique permettent le développement de l'empathie chez l'enfant. Ils apprennent les modèles de relation (soigner/ être soigné) et la régulation des émotions au travers des expériences qu'ils vivent (Sroufe, 1983).

Les variations de la qualité de l'attachement sont des conditions initiales qui, dans un contexte où les facteurs biologiques et environnementaux viennent s'additionner, jouent un rôle dynamique dans le dévelop- 
pement du fonctionnement socio-émotionnel de l'individu. Les enfants ne sont pas invulnérables, bien qu'ils ne restent pas déterminés à jamais par des patterns relationnels précoces.

L'évaluation de l'attachement nous éclaire sur les effets chez les enfants d'expériences précoces associées aux soins quotidiens hors de la maison (p. ex.: crèche), séparation (p. ex.: hospitalisation) et de perturbations significatives de prestation de soins (p. ex.: adoption et familles d'accueil). D'un côté, il se vérifie que des soins en crèche sont moins nocifs s'ils sont à temps partiel et de bonne qualité (c.-à-d.: peu d'enfants pour un même éducateur, continuité des figures au fil du temps et besoins physiques et émotionnels sécures; Sroufe et Ward, 1984). Par contre, les soins hors de la maison à plein temps et inadaptés, particulièrement s'ils débutent durant la première année de vie du bébé, quand ils se combinent avec une insensibilité parentale, peuvent fragiliser les bébés et engendrer des difficultés de développement (en particulier l'agressivité); (Belsky, 1990; NICHD, 1997; 2007).

De plus, la théorie et l'évaluation de l'attachement mettent en cause les pratiques de séparation parent-enfant quand l'enfant est malade ou au cours de procédures médicales douloureuses. La présence d'un soutien de la part des figures relationnelles significatives permet aux petits enfants de combattre et de mieux récupérer du trauma et du stress.

Les perturbations significatives dans la qualité des soins sont importantes au niveau biologique, comportemental et représentationnel, même pour les petits bébés. Le moment, la qualité du soin, le nombre d'expériences préalables et les relations de soutien, sont des variables critiques pour la détermination des effets de ces perturbations. Cependant, l'intervention ou le soutien ne se montrent jamais inutiles et ne sont jamais trop tardifs.

\section{Notes}

5 L'attachement est un type spécifique de lien appartenant à un ensemble plus vaste de liens que Bowlby et Ainsworth ont désignés par le terme de «liens affectifs». D'après Ainsworth (1985), le concept d'attachement peut être défini par un ensemble de critères, à savoir: a) il est persistant et non transitoire; b) il concerne une figure spécifique et reflète le besoin de proximité d'un individu envers un autre; c) il traite d'une relation émotionnellement significative; d) l'individu désire maintenir la proximité ou le contact avec cette figure, encore que cela puisse varier en fonction de divers facteurs, comme l'âge, l'état de l'individu ou les conditions du milieu; e) l'individu sent une certaine perturbation face à une situation de séparation involontaire et surtout quand il désire cette proximité et que celà n'est pas possible; f) l'individu recherche de sécurité et de réconfort dans la relation avec cette personne. Ce dernier critère est déterminant pour la distinction entre «liens parentaux» et «attachement des enfants aux parents» (Cassidy, 1999).

developmental phases of attachment's relationships and the research related to the factors influencing their organisational differences. From a developmental point of view, the association between attachment and psychopathology will also be explored. We will conclude with a short revision of the clinical implications of attachment theory for the paediatric practice.

\section{Key words}

Attachment.

Development.

Pediatrics. 
6 D'après Soares (1996), I'utilisation, par l'enfant, de la figure d'attachement comme «havre de sécurité" n'implique pas l'expérience de peur ou de menace chez l'enfant, alors que rechercher la mère ou rester auprès d'elle comme dans un "refuge ", signifie que l'enfant est au moins un peu alarmé. $\mathrm{Si}$, après avoir trouvé ce refuge, l'enfant parvient à se sentir rassuré, à même de retourner à l'exploration, alors la figure d'attachement devient une «base de sécurité".

7 Expliquant de façon conceptuelle le système d'attachement comme séparé du système d'exploration, Bowlby considérait toutefois leurs fonctionnements comme étant interdépendants. La qualité de l'exploration a été définie pour la première fois par Main, en 1973, comme associée à une plus longue durée des périodes de concentration de l'enfant pendant les situations de jeu avec de nouveaux jouets. Cette capacité de concentration pendant l'exploration parait dépendre a) de la capacité d'organiser les émotions et les comportements de façon intéressée et soigneuse comme réponse aux évènements "curieux", et b) de la confiance dans la disponibilité et dans l'aide prodiguée par la figure d'attachement, si l'enfant en a besoin (Grossman, Grossman, et Zimmerman, 1999).

8 Une autre caractéristique importante de cette phase concerne la capacité progressive qu'a l'enfant d'accepter la séparation plus prolongée d'avec la figure d'attachement. Son sentiment de sécurité paraît intimement lié à l'accessibilité des parents, bien que cela permette paradoxalement une plus grande capacité à tolérer leur distance (Parkers et Stevenson-Hinde, 1982). Ce changement n'implique pas l'atténuation de l'attachement mais amène des modifications des facteurs qui activent le système d'attachement (Marvin, 1977). A mesure que le modèle représentationnel de la figure d'attachement s'élabore au décours de l'expérience et se consolide, l'enfant devient capable de tolérer des suspensions dans la présence effective de cette figure, pour des périodes progressivement plus longues, sans souffrance significative. Ainsi, les séparations sont de mieux en mieux acceptées et leurs raisons sont comprises (Ainsworth et al., 1978).

9 On peut définir la sensibilité maternelle comme la capacité de la figure maternelle de prodiguer des soins inducteurs du sentiment de sécurité, c'est-à-dire des soins sensibles et adéquats. Elle s'associe à sa capacité de comprendre les caractéristiques individuelles du bébé, acceptant ses traductions comportementales. II s'agit alors d'être capable d'orchestrer des interactions harmonieuses, surtout lors des moments de stress, et ce, de manière relativement consistante (Belsky, 1999).

10 L'«attachement Q-Sort» (AQS), développé par Waters et Deane (1985) fournit une alternative à l'évaluation en laboratoire. L'AQS consiste en 90 items conçus pour discerner une série de dimensions qui reflètent le phénomène de base de sécurité chez des enfants d'âges compris entre 12 et 60 mois dans un contexte naturel (Waters, 1995). La classification peut être réalisée par des observateurs entraînés ou par les caregivers. Cette mesure comporte un indice de sécurité, la corrélation entre les classifications de l'observateur et les classifications critères. La fidélité intercoteurs est ajustée, puisqu'il a été vérifié que l'AQS est conforme à l'évaluation de la sécurité des bébés en Situation Etrange âgés de 12 à 18 mois. Cependant, les validations des classifications pour distinguer les sous-groupes d'insécure évitant, d'insécure résistant, et désorganisé/désorienté ne sont pas disponibles.

11 Certains de ces comportements pourront être observés chez les enfants ayant des perturbations neurologiques, ou avec des signes d'autisme. La distinction avec l'attachement désorganisé 
exige des précautions méthodologiques spécifiques (Barnett, Hunt, Butler, Ms Caskill, KaplanEstrin, et Pipp-Siegel, 1999).

Pour Main, le comportement parental effrayant/menaçant (p. ex. : l'abus physique) n'est pas seul à avoir des conséquences, mais aussi le comportement effrayé/menacé: "battering parents are, of course, directly frightening. There is now reason to believe, however, that frightened parental behaviour may also alarm an infant and leave him without a strategy (Main, 1990). This outcome seems especially likely if the parent withdraws from the infant as though the infant were the source of the alarm and/or appears to be in a dissociated or trancelike state »(Main, 1995, p. 426-427).

Les études de l'attachement de bébés et d'enfants avec des beoins spéciaux soutiennent aussi cette perspective qui conçoit l'attachement comme une adaptation d'origine biologique et comme un modèle de régulation dyadique, construit au fil du temps au travers de processus interactifs enfant-caregiver. Plus spécifiquement ces études ont démontré que:

- Des niveaux légèrement plus élevés d'insécurité dans l'attachement ont été observés dans les populations d'enfants avec des perturbations neurologiques (Barnett, Hunt, Butler, McCaskill, Kaplan-Estrin, et Pipp-Siegel, 1999). Nonobstant, les valeurs élevées de désorganisation trouvées dans ce type de population peuvent être liées à des questions de mesure ( $p$. ex.: ressemblances entre les symptômes neurologiques et les marqueurs de désorganisation; Pipp-Siegel, Siegel, et Dean, 1999) et pas tellement avec la qualité de la relation d'attachement avec le caregiver (Barnett et al., 1999).

- Les bébés et petits enfants porteurs de syndrome de Down (trisomie 21) atteignent les mêmes stades de développement que les enfants sains, mais à un rythme plus lent - la consolidation de la relation d'attachement se fait entre les 12 et les 24 mois (Cicchetti et Beeghly, 1990; Thompson, Cicchetti, Lamb, et al., 1985).

- En ce qui concerne les enfants autistes, les études montrent qu'ils sont capables de former des relations d'attachement avec le caregiver (Capps, Sigman, et Mundy, 1994; Rogers, Ozonoff, et Maslin-Cole, 1993; Shapiro, Sherman, Calamari, et al., 1987; Patone, et Rogers, 1984 ; Sigman, et Ungerer, 1984) et qu'environ 50\% des bébés autistes présentent des attachements sécures (Capps, Sigman, et Mundy, 1994; Shapiro, et al., 1987).

- Les études qui comparent des bébés modérément prématurés et des bébés à terme ont révélé qu'il n'existe pas de différences dans la sécurité des relations d'attachement (Rode, Chang, Niau, et al., 1981). Les exigences particulières des bébés prématurés peuvent être médiatisées par la réponse du caregiver, résultant dans le développement des patterns régulateurs adaptatifs de la dyade et donner lieu à des relations d'attachement de qualité (Cox, Hopkins, et Hans, 2000).

- Les diagnostics de Retard de Croissance (c.-à-d.: Failure to thrieve) ont été associés à des niveaux élevés d'attachement insécure (désorganisé dans les cas des plus sévères déficits du poids) en populations à risque (Crittenden, 1987; Valenzuela, 1990) ainsi que pour des échantillons de patients ambulatoires (Ward, Kessler, et Altman, 1993; Ward, Lee, et Lipper, 2000).

- Les facteurs parentaux, comme, par exemple, les conflits conjugaux, la psychopathologie maternelle (p. ex.: dépression) et le stress familial, sont des facteurs contributifs des problèmes de sommeil (Sadeh, 1996), étant donné que les mères des enfants avec des perturbations de 
sommeil sont plus à même d'exhiber des représentations distordues et détachées de leurs bébés (Benoit, Zeanah, Parker, et al., 1997) et de leurs propres expériences de soins (Benoit, Zeanah, Boucher, et al., 1992).

Soares I., Santos P., Jongenelen, et al. (1996); Soares I., Silva C., Cunha J., Costa O., Santos P. (1999a); Tereno S. (2007).

Dans une perspective de classification psychiatrique, les perturbations d'attachement dans l'enfance sont définies comme des perturbations marquées dans les relations sociales de l'enfant, commençant avant l'âge de cinq ans, qui proviennent de divers contextes sociaux et se distinguent de perturbations inhérentes au développement. Elles peuvent être de deux types: a) réponses excessivement inhibées, hyper-vigilance ou forte ambivalence à l'égard des prêteurs de soins adultes; et b) sociabilité indiscriminée (excessive sociabilité avec des étrangers) et un échec relatif à manifester des attachements sélectifs ajustés. Au-delà de ces indicateurs, le DSM-IV recherche des évidences de soins caractéristiquement pathogéniques, incluant insouciance persistante par rapport aux nécessités émotionnelles et physiques de l'enfant ou changements répétés de figure d'attachement primaire. II faut souligner que les critères de diagnostic des Perturbations d'Attachement de zéro à trois ans (NCICP, 1994) mettent l'accent sur les irrégularités de l'ambiance plutôt que sur les comportements enfantins. Toutefois, la fiabilité et la validité de ces schémas de classification ne sont pas supportées empiriquement.

Zeanah, Boris, et Lieberman (2000), afin d'élargir ces classifications psychiatriques, ont montré trois catégories de perturbations: a) perturbations de non-attachement; b) distorsion de base de sécurité; et c) perturbations d'attachements disruptives. Dans l'enchaînement du DSM-IV et de I'ICD, les perturbations relatives à la non préférence d'une figure d'attachement sont définies comme des non-attachements avec retirée émotionnelle et non-attachement avec sociabilité indiscriminée. Les distorsions de base de sécurité incluent les perturbations d'attachement avec risque pour le self (c.-à-d. : activités excessivement dangereuses ou risquées), avec attachement/inhibition (c.-à-d. : absence de comportements exploratoires), avec vigilance/hyper-soumission (c.-à-d. : émotionnellement inhibé et comportement de peur) et d'inversion de rôles (c.-à-d. : préoccupation de l'enfant pour le bien-être émotionnel de l'adulte). La perturbation d'attachement disruptif, la troisième catégorie majeure s'applique aux enfants qui ont développé une réponse de protestation, de désespoir ou non-attachement envers une séparation significative par rapport à la figure d'attachement ou une perte soudaine. Plus que n'importe quel autre diagnostic, ceux-ci sont validés par une plus grande association entre perturbations relationnelles sévères et perturbations d'attachement; d'ailleurs, seule une fidélité marginale a été démontrée (Boris, Zeanah, Larrieu, et al., 1998).

Bowlby (1958) considérait initialement que la première année de vie des enfants était une période sensible dans l'établissement de la relation d'attachement, soutenant que la carence de soins de la part d'une figure stable constitue, dans cette période, un risque pour le développement de l'enfant. Ultérieurement, l'auteur soutiendra que la période comprise entre six mois et cinq ans est aussi sensible en ce qui concerne le développement d'expectatives sur la disponibilité de(s) figure(s) d'attachement(s), encore qu'il maintienne la position selon laquelle, 
pendant les premiers six mois de vie, la plupart des bébés sont en état de grande vulnérabilité en ce qui concerne le développement du comportement d'attachement (Bowlby, 1969/1982). Quand un enfant possède plus d'une figure d'attachement, on pourrait supposer que son attachement à la figure principale pourrait être moins fort et qu'inversement, quand il possède une seule figure d'attachement, son lien à cette figure sera spécialement intense. D'après Bowlby, cette supposition n'est pas correcte: «it is a mistake to suppose that a young child diffuses his attachment over many figures in such a way that he gets along with no strong attachments to anyone, and consequently without missing any particular person when that person is away " (1969/1982, p. 308).

Reçu en septembre 2006, accepté en février 2007

\section{Remerciements}

L'élaboration de cet article a bénéficié de l'aide du laboratoire Neofarmacêutica, Lda.

Les auteurs portugais remercient le Pr Elizabeth Carlson pour avoir aimablement mis à disposition une partie de sa réflexion autour du thème de cet article, lequel a été actualisé, adapté et développé en fonction de la culture médicale portugaise et européenne.

La version française de ce manuscrit a été révisée par le Dr Fabienne Perdereau et par le Pr Antoine Guedeney auxquels nous exprimons notre profonde gratitude.

\section{Références}

[1] AGUILAR B., SROUFE L., EGELAND B. et al. : "Distinguishing the early-onset/persistent and adolescence-onset antisocial behavior types: from birth to 16 years ", Developmental Psychopathology, 2000; 12: 109-132.

[2] AINSWORTH M.: Infancy in Uganda: Infant care and the growth of love, Johns Hopkins University Press, Baltimore, 1967.

[3] AINSWORTH M.: «Object relations, dependency and attachment: a theoretical review of the infant-mother relationship ", Child Development, 1969; 40: 969-1025.

[4] AINSWORTH M.: «Attachment and dependency: a comparison», in GEWIRTZ J.L. (Ed.): Attachment and dependency, V.H. Winston, Washington, DC, 1972; p. 97-137.

[5] AINSWORTH M.: «The development of infant-mother attachment », in CALDWELL B., RICCIUTI H.N. (Ed.): Review of Child Development Research, Univ. of Chicago Press, Chicago, $1973 ;$ p. 1-94

[6] AINSWORTH M.: «Attachment across the life span », Bulletin of the New York Academy of Medicine, 1985; 61: 792-812.

[7] AINSWORTH M., BELL S.: «Mother-infant interaction and the development of competence», in CONNOLLY, BRUNNER (Eds.): The Growth of Competence, Academic Press, New York, 1974; p. 97-118.

[8] AINSWORTH M., BELL S., STAYTON D.: «Infant-mother attachment and social development: "Socialization" as a product of reciprocal responsiveness to signals ", in RICHARDS P.M. (Ed.): The integration of a child into a social world, Cambridge University Press, Cambridge, $1974 ; 99-135$. 
[9] AINSWORTH M., BLEHAR M., WATERS E., WALL S. : Patterns of attachment: A psychological study of the strange situation, Erlbaum, Hillsdale, 1978.

[10] AMERICAN PSYCHOLOGICAL ASSOCIATION: Diagnostic and Statistical Manual of Mental Disorders, 4e édition, American Psychiatric Association, Washington, DC, 1994.

[11] ANDERSON B.: "Effects of day-care on cognitive and socioemotional competence of thirteen-year-old Swedish schoolchildren ", Developmental Psychology, 1992; 63: 20-36.

[12] BARNETT D., HUNT K., BUTLER C., MSCASKILL J., KAPLAN-ESTRIN M., PIPP-SIEGEL S. : "Indices of attachment disorganization among toddlers with neurological and non-neurological problems ", in SOLOMON J., GEORGE C. (Eds.): Attachment disorganization, The Guilford Press, New York, 1999; pp. 189-212.

[13] BELSKY J.: «Developmental risks associated with infant daycare: attachment insecurity, noncompliance, and aggression ", in CHEHVAZI (Ed.): Balancing Working and Parenting, American Psychiatric Press, New York, 1990; p. 37-68.

[14] BELSKY J. : «Interactional and Contextual Determinants of Attachment Security », in CASSIDY J., SHAVER P. (Eds.): Handbook of attachment. Theory, Research and Clinical Applications, The Guilford Press, New York, London, 1999.

[15] BELSKY J., NEZWORSKI T.: Clinical Implications of Attachment, Erlbaum, Hillsdale, NJ, 1988.

[16] BENOIT D., ZEANAH C., BOUCHER C., et al. : «Sleep disorders in early childhood: association with insecure maternal attachment ", J. Am. Acad. Child Adolesc. Psychiatry, 1992; 31: 86-93.

[17] BENOIT D., ZEANAH C., PARKER K., et al.: «Working model of the child interview: infant clinical status related to maternal perceptions ", Infant Mental Health Journal, 1997; 18: 107-121.

[18] BERGER L.: From Instinct to Identity: the Development of Personality, Prentice-Hall, Englewood Cliffs, NJ, 1974.

[19] BERGER S.: University of Minnesota. Minneapolis (Manuscrit pas publié), 1994.

[20] BLEHAR M., LIEBERMAN A., AINSWORTH M.: «Early face-to-face interaction and its relation to later infant-mother attachment », Child Development, 1977; 8: 182-194.

[21] BORIS N., ZEANAH C., LARRIEU J., SCHEERINGA M., HELLER S. : «Attachment disorders in infancy and early childhood: a preliminary investigation of diagnostic criteria ", Am J Psychiatry, 1998; $155:$ 295-297.

[22] BOWLBY J.: «The nature of the child's tie to his mother », International Journal of PsychoAnalysis, 1958; 39: 350-373.

[23] BOWLBY J.: Attachment and loss: Attachment, ( $1^{\text {re }}$ et $2^{\mathrm{e}}$ édition respectivement), Basic Books, London, 1969/1982.

[24] BOWLBY J.: Attachment and loss: Separation, (1 $1^{\text {re }}$ et $2^{\mathrm{e}}$ édition respectivement), Basic Books, New York, 1973/1979.

[25] BOWLBY J.: The making and breaking of affectional bonds, Tavistock, London, 1979.

[26] BOWLBY J.: Attachment and loss: Loss, sadness and depression, Basic Books, London, 1980.

[27] BOWLBY J.: A secure base. Clinical implications of attachment theory, Routledge, London, 1988.

[28] BRAZELTON T.: Touchpoints, Guilford Press, New York, 1992.

[29] BRAZELTON T., KOSLOWSKI B., MAIN M. : "The origins of reciprocity: the early motherinput interaction ", in LEWIS, ROSENBLUM (Eds.): The effetct of Infant on its caregiver, Wiley, New York, 1974; p. 49-76.

[30] BRETHERTON I.: «Open communication and internal working models: their role in the development of attachment relationships ", in THOMPSON R. (Ed.), Socioemotional Development, University of Nebraska Press, Lincoln and London, 1990; p. 57-113.

[31] BYNG-HALL J.: «Family and couple therapy: Toward greater security ", in CASSIDY J., SHAVER P. (Eds.): Handbook of attachment: Theory, research, and clinical applications, The Guildford Press, New York, 1999; p. 625-647. 
[32] BYNG-HALL J.: «Toward a coherent story about illness and loss », in PAPADOPOULOS R., BYNG-HALL J. (Eds.): Multiple voices. Narrative in systemic family psychotherapy, Karnac, London, 2001; 103-124.

[33] CAPPS L., SIGMAN M., MUNDY P.: «Attachment security in children with autism », Developmental Psychopathology, 1994; 6: 249-261.

[34] CARLSON E.: "A prospective longitudinal study of disorganized/disoriented attachment », Child Development, 1998; 69: 1970-1979.

[35] CARLSON E., SROUFE A. : «The contribution of attachment theory to developmental psychopathology ", in CICCHETTI D., COHEN (Eds.): Theoretical Perspectives and Methodological Approaches, 1: Developlmental Processes and Psychopathology, Cambridge University Press, New York, 1995 ; p. 581-617.

[36] CARLSON E., SROUFE A.: "Reliability in attachment classification", Newsletter of the Society for Research in Child Development, 1993; 4.

[37] CARLSON V., CICCHETTI D., BURNETT D., et al. : «Disorganized/disoriented attachment relationships in maltreated infants ", Developmental Psychology, 1989; 25: 525-531.

[38] CASSIDY J.: «The nature of the child's tie ", in CASSIDY J., SHAVER P. (Eds.): Handbook of attachment: Theory, research, and clinical applications, The Guildford Press, New York, 1999: 3-20.

[39] CHISHOLM K.: "A three year follow-up of attachment and indiscriminate friendliness in children adopted from Romanian orphanages », Child Development, 1998; 69: 1090-1104.

[40] CICCHETI D., BEEGHLY M.: "An organizational approach to the study of Down syndrome: contributions to an integrative theory of development ", in CICCHETI, BEEGHLY (Eds.): Children with Down Syndrome: A developmental Perspective, Cambridge University Press, New York, 1990 ; p. 29-62.

[41] CICCHETTI D., GANIBAN J., BARNETT D.: "Contribuitions from the study of high risk populations to understanding the development of emotion regulation ", in DODGE, GARBER (Eds.): The Development of Emotion Regulation, 1991; p. 15-48.

[42] CLULOW C.: "Attachment theory and the therapeutic frame ", in CLULOW C. (Ed.), Adult attachment and couple psychotherapy, Brunner-Routledge, Sussex, 2001.

[43] COLLINS A. : Clinical relevant research on attachment and development. Communication Oral par Pr W. Andrew Collins au Departamento de Psicologia da Universidade do Minho, Portugal, avril 2005.

[44] COLLINS P., DEPUE R. : «A neurobehavioral systems approach to developmantal psychopathology: implications for disorders of affect », in CICHETTI, TOTH (Eds.): Rochester Symposium on Developmental Psychopathology (Vol.4), Erlbaum, Hillsdale, NJ, 1992 ; p. 29-101.

[45] COX S., HOPKINS J., HANS S.: «Attachment patterns in infants and their mothers: neonatal risk status and maternal representations ", Infant mental Health Journal, 2000; 21, 464-480.

[46] CRITTENDEN P.: Abusing, neglecting, problematic, and adequate dyads: differentiating by patterns of interaction, Merril-Palmer Q, 1981; 27: 201-208.

[47] CRITTENDEN P.: «Non-organic failure to thrive: deprivation or distortion », Infant Mental Health Journal, $1987 ; 8: 51-64$.

[48] CRITTENDEN P.: «Relationships at risk», in BELSKY J., NEZWORSKY T. (Eds.): Clinical implications of attachment, Lawrence Erlbaum, Hillsdale, 1988.

[49] CROKENBERG S.: «Infant irritability, mother responsiviness and social support influences on the security of infant-mother attachment ", Child Development, 1981; 52: 857-865.

[50] DALLOS R.: "Attachment narrative therapy: integrating ideas from narrative and attachment theory in systemic family therapy with eating disorders ", Journal-of-family-therapy, $2004 ; 26$ (1): 40-65.

[51] DOLAN R., ARNKOFF D., GLASS C.: «Client attachment style and the psychotherapist's interpersonal stance », Psychotherapy, 1993; 30: 408-412. 
[52] DOUGLAS J.: "Early hospital admissions and later disturbances of behavior and learning ", Dev Med Child Neurol., 1975; 17: 456-480.

[53] DOZIER M. : «Attachment organization and treatment use for adults with serious psychopathological disorders ", Development and Psychopathology, 1990; 2 : 47-60.

[54] DOZIER M., CUE K.L., BARNETT L. : “Clinician as caregivers: role of attachment organization in treatment ", Journal of Consulting and Clinical Psychology, 1994; 62: 793-800.

[55] DUGGAL S., CARLSON E., SROUFE L., et al. : «Depressive symptomatology in childhood and adolescence ", Developmental Psychopathology, 2001; 13: 143-164.

[56] EASTERBROOKS M.: "Quality of attachment to mother and to father: effects of perinatal risk status ", Child Development, 1989; 60: 825-830.

[57] EGELAND B., SROUFE A.: "Developmental sequelae of maltreatment in infancy", in CICHETTI, RIZLEY (Eds.): New Aproaches to Child Maltreatment, Jossey Bass., San Francisco, CA, 1981; 44-52.

[58] EGELAND B., FARBER E. : «Infant-mother attachment: factors related to its development and changes over time ", Child Development, 1984; 55: 753-771.

[59] EGELAND B., WEINFIELD N., BOSQUET M., et al. : «Remembering, repeating, and working through: lessons from attachment-based interventions", in OSOFSKY, FITZGERALD (Eds.): WAIMH Handbook of Infant Mental Health, Wiley, New York, 2000; p. 37-89.

[60] EMDE R.: «The infant's relationship experience: developmental and affective aspects", in SAMEROFF, EMDE (Eds.): Relationship disturbances in early childhood: a developmental approach, Basic Books, New York, 1989; p. 70-94.

[61] ERICKSON M., EGELAND B., SROUFE A.: «The realtionship between quality of attachment and behavior problems in preschool in a high risk sample", Monogr Soc Res Child Dev, $1985 ; 50: 147-186$.

[62] FEIGELMAN W., SILVERMAN A.: Chosen Children: new patterns of adoptive relationships, Praeger, New York, 1983.

[63] FISHER P., GUNNAR M., CHAMBERLAIN P., et al.: «Preventive intervention for maltreated preschool children: impact on children's behavior, neuroendocrine activity, and foster parent functioning ", J Am Acad Child Adolesc Psychiatry, 2000; 39: 1356-1364.

[64] FONAGY P.: Attachment theory and psychoanalysis, Other Press, New York, 2001.

[65] GREENBERG, M., SPELTZ, M., DEKLYEN, M., et al. : «Attachment security in preschoolers with and withouth externalizing behavior problems: replication ", Developmental Psychopathology, 1991 ; 3 : 413-430.

[66] GREENOUGH W., BLACK J.: «Induction of brain structure by experience: substrates for cognitive development », in GUNNAR, NELSON (Eds.) : Developmental Behavioral Neuroscience: The Minnesota Symposium on Child Psychology (Vol. 24), Lawrence Erlbaum Associates, Hillsdale, NY, 1992; p. 155-200.

[67] GROSSMANN K., GROSSMANN K.E., HUBER F., et al.: "German children's behavior towards their mothers at 12 months and their fathers at 18 months in Ainsworth's strange situation ", Int J Behav Dev., 1981; 4: 157-181.

[68] GROSSMANN K., GROSSMANN K.E., SPANGLER G., et al.: "Maternal sensitivity and newborn orienting responses as related to quality of attachment in Northern Germany ", Monogr Soc Res Child Dev., 1985; 50: 233-256.

[69] GROSSMAN K.E., GROSSMAN K., ZIMMERMAN P.: «A wider view of attachment and exploration: stability and change during the years of immaturity", in CASSIDY J., SHAVER P. (Eds.): Handbook of attachment. Theory, Research and Clinical Applications, The Guilford Press, New York, London, 1999.

[70] GUIDANO V.: The complexity of the self: A developmental approach to psychopathology and therapy, Guilford, New York, 1987.

[71] GUIDANO V.: The self in process. Toward a post-rationalistic cognitive therapy, Guilford, New York, 1991. 
[72] GUNNAR M. : «Early adversity and the development of stress reactivity and regulation », in NELSON C. (Ed.): The Effects of Adversity on Neurobehavioral Development, 31. Minnesota Symposia on Child Psychology, Lawrence Erlbaum, Mahwah, NJ, 2001; p. 163-200.

[73] GUNNAR M., MANGELSDORF S., LARSON M., HERTSGAARD L. : «Attachment, temperament, and adrenocortical activity in infancy. A study of psychoendocrine regulation ", Developmental Psychology, 1989; 25: 355-363.

[74] HARLOW H.: «The maternal affectional system ", in FOSS (Ed.): Determinants of Infant Behavior, Wiley, New York, 1963; p. 3-29.

[75] HARLOW H., HARLOW M.: “The affectional systems", in SCHRIER, HARLOW, STOLLNITZ (Eds.): Behavior of Nonhuman Primates, Academic Press, New York, 1965; p. 1-28.

[76] HOLMES J.: The search for the secure base. Attachment theory and Psychotherapy, Brunner-Routledge, Sussex, 2001.

[77] HRDY S.: Mother Nature: A history of mothers, infants, and natural selection, Ballatine Books, New York, 2000.

[78] JACOBITZ D., HAZEN N., RIGGS S. : «Disorganized mental processes in mothers, frightened/frightening caregiving, and disorganized/disoriented behavior in infancy». Presented at the Society for Research in Child Development, Washington, DC, April 1997.

[79] JANG S., SMITH S., LEVINE T. : «To Stay or To Leave? The Role of Attachment Styles in Communication Patterns and Potential Termination of Romantic Relationships Following Discovery of Deception », Communication Monographs, 2002; 69: 236-252.

[80] KALER S., FREEMAN B.: «Analysis of environmental deprivation: cognitive and social development in Romanian orphans ", J Child Psychol Psychiatry, 1994 ; 35 : 759-781.

[81] KOBAK R., SHAVER P.: Strategies for maintaining felt security: A theoretical analysis of continuity and change in styles of social adaptation, University of Denver, 1987.

[82] KOOP C.: «Antecedents of self-regulation: a developmental view », Developmental Psychology, 1982 ; 18: 199-214.

[83] KORFMACHER J., ADAM E., OGAWA J., EGELAND B.: «Adult attachment: implications for the therapeutic process in a home visitation intervention ", Applied Developmental Science, 1997 ; 1 : 43- 52.

[84] KRAEMER G.: «A psychobiological theory of attachment», Behav Brain Sci, 1992; 15: 493-541.

[85] LIEBERMAN A.F., WESTON D., PAWL J.H.: «Preventive intervention and outcome with anxiously attached dyads ", Child Development, 1991; 62: 199-209.

[86] LIOTTI G.: «Disorganized/disoriented attachment in the etiology of the dissociative disorders ", Dissociation, $1992 ; 4$ : 196-204.

[87] LIOTTI G.: «Disorganized attachment, models of borderline states and evolutionary psychotherapy ", in GILBERT P., BAILEY K. (Eds.): Genes on the couch. Explorations in evolutionary psychotherapy, Brunner-Routledge, Philadelphia, 2000.

[88] LIOTTI G. : «Disorganization of attachement as a model for understanding dissociative psychopathology ", in SOLOMON J., GEORGE C. (Eds): Attachment Disorganisation, The Guildford Press, NY, 2005; p. 291-317.

[89] LOJKASEK M., COHEN N., MUIR E. : "Where is the infant in infant intervention? A review of the literature on changing troubled mother-infant relationships ", Psychoterapy, 1994; 31 : 208-220.

[90] LYONS-RUTH K., REPACHOLI B., MCLEOD S. et al. : «Disorganized attachment behavior in infancy: short-term stability, maternal and infant correlates and risk-related subtypes ", Developmental Psychopathology, 1991; 3: 377-396.

[91] LYONS-RUTH K., BRONFMAN E., PARSONS E. : « Maternal disrupted affective communication, maternal frightened or frightening behavior, and disorganized infant attachment strategies ", Monogr Soc Res Child Dev, 1999; 64: 67-96.

[92] MAIN M.: «Play, exploration, and competence as related to child-adult attachment», John Hopkins University, thèse de Doctorat non publiée, 1973. 
[93] MAIN M. : «Analysis of a peculiar form of reunion behavior seen in some daycare children: its history and sequelae in children who are home-reared ", in WEBB R. (Ed.): Social development in day care, John Hopkins University Press, Baltimore, 1977.

[94] MAIN M. : «Avoidance in the service of attachment: a working paper », in IMMELMAN K., BARLOW G., PETRINOVICH L., MAIN M. (Eds.): Behavioral development: The Bielefeld interdisciplinary program, Cambridge University Press, Cambridge, England, 1981; p. 651-693.

[95] MAIN M.: «Cross-cultural studies of attachment organization: Recent studies, changing methodologies, and the concept of conditional strategies ", Human Development, 1990; 33: 48-61. [96] MAIN M.: «Attachment: Overview with implications for clinical work », in GOLDBERG S., MUIR R., KERR J. (Eds.): Attachment theory: social development, and clinical perspectives, Analytic Press, Hillsdale, NJ, 1995; p. 407-474.

[97] MAIN M., WESTON D.: «The quality of the toddler's relationship to mother and father: related to conflict behavior and the readiness to establish new relationships ", Child Development, $1981 ; 52:$ 932-940.

[98] MAIN M., CASSIDY J.: «Categories of response to reunion with the parent at age 6: Predictable from infant attachment classifications and stable over a 1-month period", Developmental Psychology, 1988; 24: 1-12.

[99] MAIN M., HESSE E.: «Parents' unresolved traumatic experiences are related to infant disorganized attachment status: Is frightening and/or frightening parental behavior the linking mechanism? ", in GREENBERG M., CICCHETTI D., CUMMINGS E. (Eds.): Attachment in the preschool years, Chicago University Press, Chicago, 1990; p. 161-182.

[100] MAIN M., SOLOMON J.: «Procedures for classifying infants as disorganized/disoriented during the Ainsworth Strange Situation? ", in GREENBERG M., CICCHETTI D., CUMMINGS E. (Eds.): Attachment in the preschool years, Chicago University Press, Chicago, 1990; p. 121-160. [101] MAIN M., MORGAN H.: «Disorganization and disorientation in infant strange situation behavior: phenotypic resemblance to dissociative states ", in Michelson L., Ray W. (Eds.): Handbook of dissociation: Theoretical, empirical, and clinical perspectives, Plenum Press, New York, 1996; 107-138.

[102] MANGELDORF S., GUNNAR M., KESTENBAUM R., et al. : «Infancy proneness-to-distress temperament, maternal personality, and mother-infant attachment: associations and goodness of fit ", Child Development, 1990; 61: 820-831.

[103] MARCOVITCH S., GOLDBERG S., GOLD A., et al.: "Determinants of behavioral problems in Romanian children adopted in Ontario ", Int J Behav Dev, 1997; 20: 17-31.

[104] MARVIN R. : «An ethological cognitive model for the attenuation of mother-child attachment behavior ", in ALLOWAY T., KRAMER L., PLINER P. (Eds.): Advancesin the study of communication and effect, vol. 3: The development of social attachments, Plenum Press, New York, 1977.

[105] NCICP: Diagnostic Classification: 0-3, Zero-to-Three, National Center for Infant Clinical Programs, Arlington, VA, 1994.

[106] NICHD Early Child Care Research Network: "The effects of infant child care on infantmother attachment security: results of the NICHD study of early child care ", Child Development, 1997; 68: 925-933.

[107] NICHD Early Child Care Research Network: «Psicopatologia do Desenvolvimento", Communication Oral par Prof. Jay Belsky au Departamento de Psicologia da Universidade do Minho, Portugal, Janvier 2007.

[108] NORHAUS B., SOLNIT A.: Zero to three, National Center for Clinical Infant Programs Washington, DC, $1990 ; 1-4$.

[109] O'CONNOR M., SIGMAN M., BRILL N.: «Disorganization of attachment in relation to maternal alcohol consumption ", J Consult Clin Psychol, 1987; 55 : 831-836.

[110] O'CONNOR T., BREDENKAMP D., RUTTER M., THE ENGLISH AND ROMANIAN ADOPTEES (ERA) STUDY TEAM: «Attachment disturbances and disorders in children exposed to early severe deprivation », Infant Mental Health Journal, 1999; 20: 10-29. 
[111] OGAWA J., SROUFE L., WEINFIELD N., et al. : "Development and the fragmented self: a longitudinal study of dissociative symptomatology in a non-clinical sample ", Developmental Psychopathology, 1997; 4: 855-879.

[112] PARKES C., STEVENSON-HINDE J.: The place of attachment in human behavior, Tavistock Publications, London, 1982.

[113] PATONE J., ROGERS S.: "Attachment characteristics of autistic and PDD preschoolaged children". Presented at the 3rd Biennial Developmental Psychology Research Group Retreat, Estes Park, CO, May 1984.

[114] PEDERSON D.R., GLEASON K.E., MORAN G., BENTO S. : « Maternal attachment representations, maternal sensitivity, and the infant-mother attachment relationship ", Developmental Psychology, 1998; 34 (5): 925-933.

[115] PIANTA R., EGELAND B., SROUFE A. : «Maternal stress in children's development: predictions of school outcomes and identification of protective factors ", in ROLF, MASTEN, CICCHETI, et al. (Eds.) : Risk and Protective Factors, Plenum Publishers, New York, 1990; 215-235. [116] PIPP-SIEGEL S., SIEGEL C., DEAN J.: « Neurological aspects of the disorganized/disoriented attachment classification system: differenciating quality of the attachment relationship from neurological impairment ", Monogr Soc Res Child Dev, 1999; 64 : 25-44.

[117] POSADA G., WATERS E., CROWELL J., et al.: «Is it easier to use a secure mother as a secure base? Attachment Q-sort correlated of the Berkeley Adult Attachment Interview ", Monogr Soc Res Child Dev, 1995; 48: 133-145.

[118] POSADA G., JACOBS A., CARBONELL O., et al.: " Maternal care and the attachment security in ordinary and emergency contexts ", Developmental Psychology, 1999; 34: 925-933. [119] Putnam F.: «Dissociation and disturbances of self », in CICCHETTI, TOTH (Eds.): Disorders and Dysfunctions of the Self (5), 251-265. Rochester Symposium on Developmental Psychopathology, Rochester University Press, Rochester, NY, 1994.

[120] QUINTON D., RUTTER M.: «Early hospital admissions and later disturbances of behavior ", Dev Med Child Neurol, 1976; 18: 447-459.

[121] RADKE-YARROW M., CUMMINGS E., KUCZYNSKI L., CHAPMAN M.: «Patterns of attachment in two-and-three-years-olds in normal families and families with parental depression ", Child development, 1985; 56: 884-893.

[122] RENKEN B., EGELAND B., MARVINNEY D., MANGELSDORF S., SROUFE A.: «Early childhood antecedents of aggression and passive withdrawal in early elementary school ", Journal of Personality, 1989; 57: 257-281.

[123] ROBERTSON J. : A two-year old goes to hospital (film), Penn State Audio-Visual Services, University Park, PA, 1953.

[124] ROBERTSON J., ROBERTSON J. : "Young children in brief separation: a fresh look», Psychoanalytic Study of the child, $1971 ; 26: 264-315$.

[125] ROBINSON J.: "Attachment Problems and Disorders in infants and Young Children: Identification, Assessment, and Intervention ", Inf Young Children, 2002; 14: 6-18.

[126] RODE S., CHANG P., NIAU P., et al. : «Attachment patterns in infants separated at birth », Developmental Psychology, 1981; 17: 188-191.

[127] RODNING C., BECKWITH L., HOWARD J.: "Quality of attachment and home environments in children prenatally exposed to PCP and cocaine ", Developmental Psychopathology, $1991 ; 3: 351-366$.

[128] ROGERS S., OZONOFF S., MASLIN-COLE C.: «Developmental aspects of attachment behavior in young children with pervasive developmental disorders ", J Am Acad Child Adolesc Psychiatry, 1993; 32 : 1274-1282.

[129] RUTTER M. : Maternal deprivation reassessed, $2^{\mathrm{e}}$ édition, Penguin, London, 1981.

[130] SADEH A.: "Stress, trauma, and sleep in children », Child Adolesc Psychiatr Clin N Am, 1996 ; 5 : 685-700. 
[131] SAMEROFF A.J., EMDE R.: Relationship disturbances in early childhood: A developmental approach, Basic Books, New York, 1989.

[132] SANDER L. : «Infant and caretaking environment», in ANTONY (Ed.): Explorations in Child Psychiatry, Plenum, New York, 1975; p. 129-166.

[133] SCHNEIDER-ROSEN K., BRAUNWALD K., CARLSON V., CICHETTI D.: «Current perspectives in attachment theory: illustration from the study of maltreated infants ", Monogr Soc Res Child Dev, 1985; 50 : 194-210.

[134] SCHUENGEL C., BAKERMANS-KRANENBURG ??, VAN IJZENDOORN M., BLOM M.: "Unresolved loss and Infant disorganisation. Links to frightening maternal behavior ", in SOLOMON J. GEORGE C. (Eds) : Attachment Disorganisation, The Guildford press, New York, 1999; p. 71-94.

[135] SCHUENGEL C., VAN IJZENDOORN M. : “Guest editors' introduction to the special issue: Attachment in mental health institutions ", Attachment \& Human Development, 2001 ; 3 (3): 239242.

[136] SHAPIRO T., SHERMAN M., CALAMARI G., et al.: "Attachment in autism and other developmental disorders », J Am Acad Child Adolesc Psychiatry, 1987; 26: 480-484.

[137] SHORE A.: Affect regulation and the origin of the self. The neurobiology of emotional development, Erlbaum, Mahwah, NJ, 1994.

[138] Siegel D.: «Toward an interpersonal neurobiology of the developing mind: Attachment relationships, "mindsight", and neural integration ", Infant Mental Health Journal, 2001; 22 : 1-2, 67-94.

[139] SIGMAN M., UNGERER J.: «Attachment behaviors in autistic children », J Autism Dev Disord, $1984 ; 14: 231-243$.

[140] SINGER L., BRODZINSKY D., RAMSAY D., STEIR M., WATERS E. : « Mother-infant attachment in adoptive families ", Child Development, 1985; 56: 1543-1551.

[141] SLADE A. : "Attachment theory and research: implications for the theory and practice on individual psychotherapy with adults ", in CASSIDY J., SHAVER P. (Eds.) : Handbook of attachment: Theory, research and clinical applications, The Guilford Press, NY, 1999; p. 575-594.

[142] SOARES I.: Representação da vinculação na idade adulta e na adolescência. Estudo intergeracional: mãe-filho(a). Braga: Centro de Estudos em Educação e Psicologia, Instituto de Educação e Psicologia, Universidade do Minho, 1996, (2édition en 2000).

[143] SOARES I. : «A vinculação vinculada. Lição Síntese », Departamento de Psicologia, Instituto de Educação e Psicologia, Universidade do Minho (Manuscrit non publié), 2002.

[144] SOARES I., SANTOS P., JONGENELEN I., HENRIQUES M., SILVA A., et al. : "Avaliação da vinculação e da regulação autonómica da frequência cardíaca na Situação Estranha ", in ALMEIDA L., ARAÚJO S., et al. (Org.): Avaliação Psicológica: Formas e Contextos, IV Vol. Braga: APPORT, 1996.

[145] SOARES I., SILVA C., CUNHA J., COSTA O., SANTOS P.: «Attachment organizations and heart-rate variability in the Strange Situation. Aqui e além do cérebro ", Actas do $2^{\circ}$ Simpósio da Fundação Bial, Fundação Bial, Porto, 1999; p. 285-291.

[146] SPANGLER G., GROSSMAN K.E.: «Biobehavioral organization in securely and insecurely attached infants ", Child Development, 1993; 64: 1439-1450.

[147] SPITZ R.: «Anaclitic depression: An inquiry into the genesis of psychiatric conditions in early childhood - II ", The Psychoanalytic Study of the Child, 1946; 2: 313-342.

[148] SROUFE A. : «Infant-caregiver attachment patterns of adaptation in preschool: the roots of maladaptation and competence ", in PERLMUTTER M. (Ed.): Minnesota symposium in child psychology, Lawrence Erlbaum, Hillsdale, 1983.

[149] SROUFE A. : "Bowlby's contribution to psychoanalytic theory and developmental psychology ", Journal of Child Psychology and Psychiatry, 1986; 27: 841-849.

[150] SROUFE A. : «Relationships, self, and individual adaptation », in SAMEROFF, EMDE (Eds.): Relationship Disturbances in Early Childhood: A Developmental Approach, Basic Books, New York, 1989; 70-94. 
[151] SROUFE A. «An organizational perspective on the self », in CICHETTI D., BEEGHLY M. (Eds.): The self in transition: infancy to childhood, University of Chicago Press, Chicago, 1990; p. 281-307.

[152] SROUFE A.: Emotional Development: The Organization of Emotional Life in the Early Years, Cambridge University Press, New York, 1995.

[153] SROUFE A., WATERS E. : «Attachment as an organizational construct», Child Development, 1977; 48: 1184-1199.

[154] SROUFE L., WARD M.: "The importance of early care", in QUARM, BORMAN, GIDEONESE (Eds.): Women in the Workplace: the effects on Families, Ablex, Norwood, 1984 ; p. 35-60 [155] SROUFE A., EGELAND B., KREUTZER T.: «The fate of early experience following developmental change: Iongitudinal approaches to individual adaptation in childhood ", Child Development, $1990 ; 61$ : 1363-1373.

[156] SROUFE A., CARLSON E., LEVY A., EGELAND B. : «Implications of attachment theory for developmental psychopathology ", Development and Psychopathology, 1999 ; 11 (1): 1-14. [157] SROUFE A., DUGGAL S., WEINFIELD N., CARLSON E.: «Relationships, Development and Psychopathology ", in SAMEROFF A.J., LEWIS M., MILLER S.M. (Eds): Handbook of Developmental Psychopathology, Kluwer Academic/Plenum Publishers, New York, 2000.

[158] STERN D.: "The goal of structure of mother and infant play", Journal American Academy Child Psychiatry, 1974; 13: 402-421.

[159] STERN D.: The Motherhood Constellation: A Unified View of Parent-Infant Psychotherapy, Basic Books, New York, 1995.

[160] STOVALL K., DOZIER M.: «The development of attachment in new relationships: Single subject analyses for 10 foster infants », Development and Psychopathology, 2000 ; 12 (2): 133-156. [161] SUSMAN-STILLMAN A., KALKOWSKE M., EGELAND B. : «Infant temperament and maternal sensitivity as predictors of attachment security ", Infant Behavior Development, 1996; 52, 857-865.

[162] TERENO S.: «Ontogenia da Vinculação Humana: Vinculação na Primeira Infância. Departamento de Psicologia, Instituto de Educação e Psicologia », Universidade do Minho (Manuscrit non publié), 2004.

[163] TERENO S.: «Intergeracionalidade da Vinculação: o papel da conjugalidade ». Thèse de Doctorat. Departamento de Psicologia, Instituto de Educação e Psicologia, Universidade do Minho (Manuscrit non publié), 2007.

[164] THOMPSON R., CICCHETTI D., LAMB M., et al.: "The emotional responses of Down syndrome and normal infants in the Strange Situation: the organization of affective behavior in infants ", Developmental Psychopathology, 1985; 21 : 828-831.

[165] TIZARD B.: Adoption: A second chance, The Free Press, New York, 1977.

[166] TIZARD B., HODGES J.: «The effect of early institucional rearing on the development of eight-year-old children ", J Child Psychol Psychiatry, 1978; 19: 99-118.

[167] VALENZUELA M.: «Attachment in chronically underweight young children », Child Development, $1990 ; 1: 1984-1996$.

[168] VAN IJZENDOORN M., SAGI A.: «Cross-cultural patterns of attachment: universal and contextual dimension ", in CASSIDY J., SHAVER P. (Eds.): Handbook of attachment: Theory, research and clinical applications, The Guilford Press, New York, 1999; 713-734.

[169] VANDEL D., CORASANITI M.: «Child care and the family: complex contributors to child development ", in MCCARTNEY (Ed.): Child Care and Maternal Employment: A Social Ecology Approach, 49, 23-37, Jossey-Bass, San Francisco, CA, 1990.

[170] VAUGHN B., WATERS E.: «Attachment Behavior at home and in the laboratory: Q-sort observations and strange situationclassifications of one-year-olds ", Child Development, 1990; 61, 1965-1973.

[171] VAUGHN B., BOST K.: “Attachment and Temperament ", in CASSIDY, SHAVERS (Eds.): Handbook of Attachment theory research and clinical Applications, The Guildford Press, New York, 1999 ; p. 198-225. 
[172] WARD M., KESSLER D., ALTMAN S. : «Infant-mother attachment in children with failure to thrive ", Infant Mental health Journal, 1993; 14: 208-220.

[173] WARD M., LEE S., LIPPER E. : «Failure-to-thrive is associated with dosorganized infantmother attachment and unresolved maternal attachment ", Infant Mental health Journal, 2000; 21: 428-442.

[174] WATERS E. : “The attachment Q-set », in WATERS E., VAUGHN B., POSADA G., KONDOIKEMURA K. (Eds.): Caregiving, cultural, and cognitive perspectives on secure-base behavior and working models. New growing points of attachment theory and research. Monographs of the Society for Research in Child Development, 1995; serial n² 244, vol. 60, n² 2-3: 247-254.

[175] WATERS E., DEANE K.: «Defining and assessing individual differences in attachment relationships: Q-methodology and the organization of behavior in infancy and early childhood ", in BRETHERTON I., WATERS E. (Eds.): Growing points on attachment theory and research. Monographs of the Society for Research in Child Development, 1985; 50 (Serial N²09).

[176] WHO: International Classification od Diseases: Clinical Descriptions and Diagnostic Guidelines, $10^{e}$ édition, World Health Organization, Geneva, 1992.

[177] ZEANAH C., BORIS N., LIEBERMAN A. : «Attachment disorders in infancy ", in LEWIS, SAMEROFF (Eds.): Handbook of Developmental Psychopathology, Basic Books, New York, 2000 ; p. 293-307. 